\title{
Molecular gas chemistry in AGN
}

\section{The IRAM 30 m survey of NGC 1068}

\author{
A. Usero ${ }^{1,2}$, S. García-Burillo ${ }^{1}$, A. Fuente ${ }^{1}$, J. Martín-Pintado ${ }^{3}$, and N. J. Rodríguez-Fernández ${ }^{4}$ \\ 1 Observatorio Astronómico Nacional (OAN), C/ Alfonso XII 3, 28014 Madrid, Spain \\ 2 Instituto de Matemáticas y Física Fundamental, CSIC, C/ Serrano 113bis, 28006 Madrid, Spain \\ 3 Instituto de Estructura de la Materia, DAMIR-CSIC, C/ Serrano 121, 28006 Madrid, Spain \\ ${ }^{4}$ LERMA (UMR 8112), Observatoire de Paris, 61 Av. de l'Observatoire, 75014 Paris, France
}

Received 1 December 2003 / Accepted 5 February 2004

\begin{abstract}
There is observational evidence that nuclear winds and X-rays can heavily influence the physical conditions and chemical abundances of molecular gas in the circumnuclear disks (CND) of Active Galactic Nuclei (AGN). In this paper we probe the chemical status of molecular gas in the CND of NGC 1068, a prototypical Seyfert 2 galaxy. Precedent claims that the chemistry of molecular gas in the nucleus of NGC 1068 is abnormal by galactic standards were based on the high $\mathrm{HCN} / \mathrm{CO}$ luminosity ratio measured in the CND. Results from new observations obtained in this survey have served to derive abundances of molecular species such as $\mathrm{SiO}, \mathrm{CN}, \mathrm{HCO}^{+}, \mathrm{HOC}^{+}, \mathrm{H}^{13} \mathrm{CO}^{+}$and $\mathrm{HCO}$. These estimates are complemented by a re-evaluation of molecular abundances for $\mathrm{HCN}, \mathrm{CS}$ and $\mathrm{CO}$, based on previously published single-dish and interferometer observations of NGC 1068. We report on the first detection of SiO emission in the CND of NGC 1068. The estimated large abundance of $\mathrm{SiO}$ in the $\mathrm{CND}, X(\mathrm{SiO}) \sim(5-10) \times 10^{-9}$, cannot be attributed to shocks related to star formation, as there is little evidence of a recent starburst in the nucleus of NGC 1068. Alternatively, we propose that silicon chemistry is driven by intense X-ray processing of molecular gas. We also report on the first extragalactic detection of the reactive ion $\mathrm{HOC}^{+}$. Most remarkably, the estimated $\mathrm{HCO}^{+} / \mathrm{HOC}^{+}$abundance ratio in the nucleus of NGC 1068, 30-80, is the smallest ever measured in molecular gas. The abundances derived for all molecules that have been the subject of this survey are compared with the predictions of models invoking either oxygen-depletion or X-ray chemistry in molecular gas. Our conclusions favour an overall scenario where the CND of NGC 1068 has become a giant X-ray Dominated Region (XDR).
\end{abstract}

Key words. galaxies: individual: NGC 1068 - galaxies: Seyfert - galaxies: nuclei - galaxies: ISM - ISM: abundances radio lines: galaxies

\section{Introduction}

Active Galactic Nuclei (AGN) are able to inject vast amounts of energy into their host galaxies, carried by strong radiation fields and rapidly moving jets. It is predictable that AGN should have a disruptive influence on the gas reservoir near their central engines. There is multi-wavelength observational evidence that the general properties of neutral interstellar matter in AGN differ from those of quiescent star-forming disks and starburst galaxies (Genzel et al. 1998; Laurent et al. 2000). In particular, molecular gas close to the central engines of active galaxies can be exposed to a strong X-ray irradiation. While the accretion disks of AGN are strong UV emitters, the bulk of the UV flux can be attenuated by neutral gas column densities of only $N(\mathrm{H}) \sim 10^{21} \mathrm{~cm}^{-2}$. Hard X-ray photons $(2-10 \mathrm{keV})$ can penetrate neutral gas column densities out to $N(\mathrm{H}) \sim 10^{23}-10^{24} \mathrm{~cm}^{-2}$, however. Therefore, X-ray dominated regions (XDR) could become the dominant sources

Send offprint requests to: A. Usero,

e-mail: antonio.u@imaff.cfmac.csic.es of emission for molecular gas in the harsh environment of circumnuclear disks (CND) of AGN, as originally argued by Maloney et al. (1996).

First observational evidence that the physical and chemical properties of molecular gas in the CND of AGN depart from "normality" came from the single-dish and interferometer observations of $\mathrm{HCN}$ and CO emission in NGC 1068 (Tacconi et al. 1994; Sternberg et al. 1994). This prototypical Seyfert 2 galaxy hosts a circumnuclear starburst ring of $\sim 2.5-3 \mathrm{kpc}$-diameter (see Fig. 1); the ring delimits a $2.3 \mathrm{kpc}$ stellar bar detected by Scoville et al. (1988) in the NIR. The strong emission detected in the 1-0 and 2-1 CO lines coming from the starburst ring corroborates that massive star formation is fed by a significant gas reservoir (Planesas et al. 1989, 1991; Helfer \& Blitz 1995; Schinnerer et al. 2000). Significant CO emission arises also from a $200 \mathrm{pc}$ CND of $M\left(\mathrm{H}_{2}\right) \sim 5 \times 10^{7} M_{\odot}$ (inferred using a $N\left(\mathrm{H}_{2}\right) / I(\mathrm{CO})$ conversion factor of $2.2 \times 10^{20} \mathrm{~cm}^{-2}\left(\mathrm{~K} \mathrm{~km} \mathrm{~s}^{-1}\right)^{-1}$, from Solomon \& Barrett 1991). The CND, partly resolved into two knots, surrounds 
$\left(0^{\prime \prime}, 0^{\prime \prime}\right)$ CND
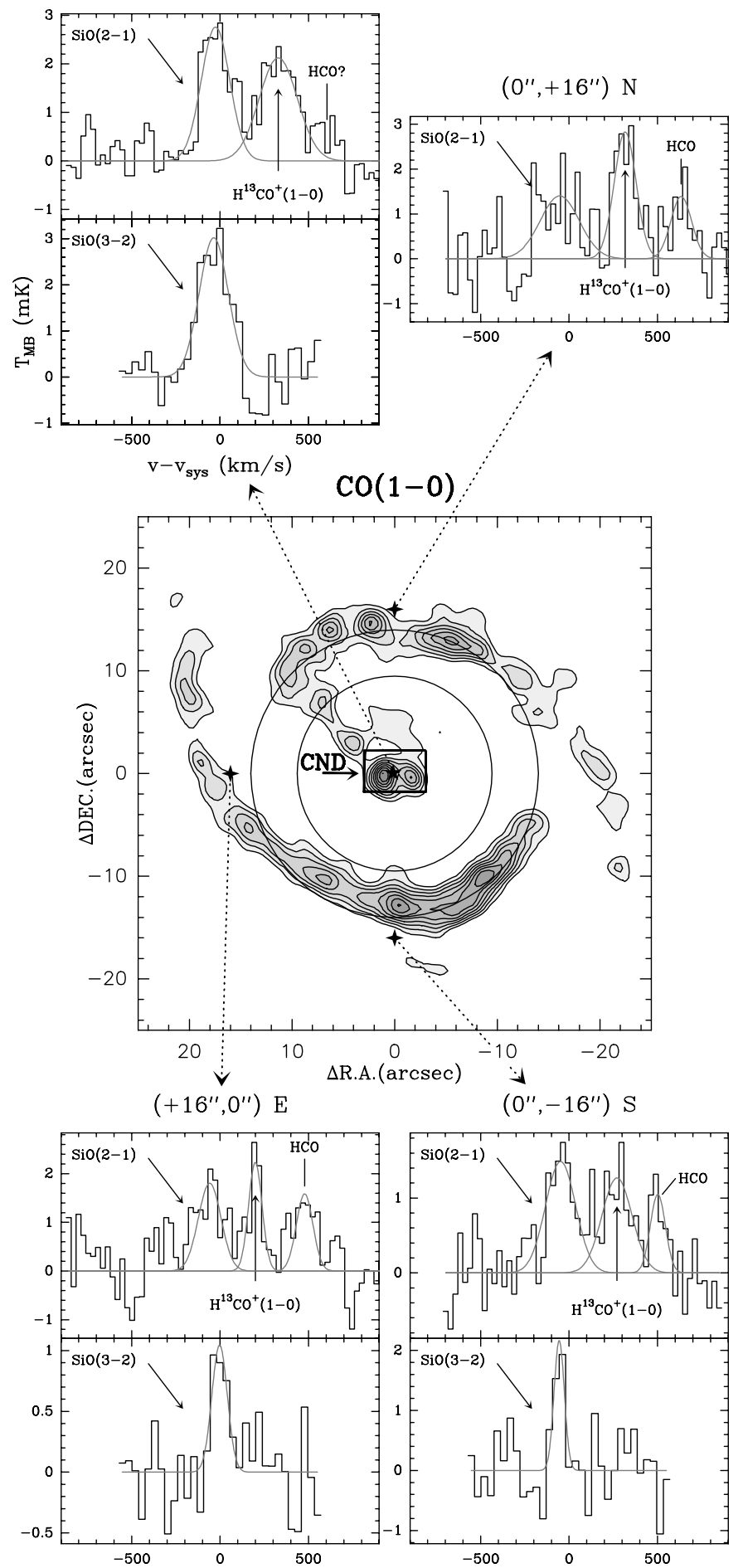

Fig. 1. Emission spectra of the 2-1 and 3-2 lines of $\mathrm{SiO}$ detected in the inner $3 \mathrm{kpc}$ of NGC 1068. Four starred markers, overlaid on the $\mathrm{CO}(1-0)$ integrated intensity map of Schinnerer et al. (2000), highlight the central positions of the beams in the disk where we searched for $\mathrm{SiO}$ emission: the central offset $\left(0^{\prime \prime}, 0^{\prime \prime}\right)$ coincides with the position of the AGN, given by the S1 compact radio-source of Gallimore et al. $(1996 \mathrm{~b})\left(\alpha_{2000}=02^{\mathrm{h}} 42^{\mathrm{m}} 40^{\mathrm{s}} \cdot 71, \delta_{2000}=-00^{\circ} 00^{\prime} 47.9^{\prime \prime}\right)$, while offsets $\mathrm{N}, \mathrm{S}$ and $\mathrm{E}$ probe the $\mathrm{SiO}$ emission over the starburst ring. Emission in the $\mathrm{H}^{13} \mathrm{CO}^{+}(1-0)$ and $\mathrm{HCO}(1-0)$ lines is detected in the $\mathrm{CND}$ and over the starburst ring. The circles represent the beam sizes at $130.3 \mathrm{GHz}\left(19^{\prime \prime}\right)$ and $86.8 \mathrm{GHz}\left(28^{\prime \prime}\right)$. the position of the active nucleus identified as the compact radio-source S1 in the map of Gallimore et al. (1996a). Most remarkably, the CND is prominent in $\mathrm{HCN}$ emission (Tacconi et al. 1994). According to the analysis of Sternberg et al. (1994), the high $\mathrm{HCN} / \mathrm{CO}$ intensity ratio measured by Tacconi et al. (1994) ( 1-10) leads to an abnormally high $\mathrm{HCN} / \mathrm{CO}$ abundance ratio in the nucleus of NGC 1068: $N(\mathrm{HCN}) / N(\mathrm{CO}) \sim$ a few $10^{-3}-10^{-2}$, i.e., the highest ratio ever found in the centre of any galaxy.

Different explanations have been advanced to quantify the possible link between the anomalous HCN chemistry and the presence of an active nucleus in NGC 1068. The selective depletion of gas-phase oxygen in the dense molecular clouds would explain the high $\mathrm{HCN}$-to-CO abundance ratio (Sternberg et al. 1994; Shalabiea \& Greenberg 1996). The same oxygen depletion scheme predicts a lower-than-normal abundance of all oxygen-bearing species. Alternatively, an increased X-ray ionization of molecular clouds near the AGN could enhance the abundance of HCN (Lepp \& Dalgarno 1996). Furthermore, X-rays could evaporate small ( $\sim 10 \AA)$ silicate grains, increasing the fraction in gas phase of all refractory elements and subsequently enhancing the abundance of some molecules (e.g., SiO) in X-ray irradiated molecular gas (Voit 1991; Martín-Pintado et al. 2000). While the aforementioned scenarios succeed to reproduce the measured enhancement of $\mathrm{HCN}$ relative to $\mathrm{CO}$ in NGC 1068, their predictions about the abundances of other molecular species differ significantly. The lack of tight observational constraints for these models, prompted by the first mm-observations made in NGC 1068, has hampered thus far the choice of an optimum scenario, however.

In this paper we discuss the results of a molecular survey made in NGC 1068 with the IRAM $30 \mathrm{~m}$ mm-telescope. NGC 1068 is the optimum target to quantify the feed-back of activity and star formation on the chemistry of molecular gas. Furthermore, the spatial resolution of the $30 \mathrm{~m}$ telescope is well suited to discern between the emission coming from the star forming ring and that coming from the CND. We discuss the results obtained from new mm-observations of 6 molecular species. The list includes: $\operatorname{SiO}(v=0, J=2-1$ and $J=3-2), \mathrm{HCO}(J=3 / 2-1 / 2, F=2-1), \mathrm{H}^{13} \mathrm{CO}^{+}(J=1-0)$, $\mathrm{HCO}^{+}(J=1-0), \operatorname{HOC}^{+}(J=1-0)$ and $\mathrm{CN}(N=2-1)$. For comparison purposes, we include in our analysis the results from previous single-dish and interferometer observations of CO $(J=1-0$ and 2-1 from Schinnerer et al. 2000; $J=4-3$ from Tacconi et al. 1994), HCN ( $J=1-0$ and 4-3) (Tacconi et al. 1994) and CS ( $J=2-1)$ (Tacconi et al. 1997). This data base has served for estimating the abundances of eight molecular species in the CND of NGC 1068 using LVG model calculations. The inferred abundances are compared with the predictions of models invoking either oxygen-depletion or X-ray chemistry in molecular gas. We present in Sect. 2 the $30 \mathrm{~m}$ observations made for this survey as well as the data compiled from previous works on NGC 1068. Section 3.1 presents the results obtained from our $\mathrm{SiO}$ study and their implications for the CND chemistry. Section 3.2 is devoted to discuss the chemistry of the $\mathrm{HOC}^{+} / \mathrm{HCO}^{+}$active ions. The molecular gas inventory of the CND is globally presented and discussed in Sect. 4. 
Table 1. Main parameters of the new $30 \mathrm{~m}$ observations (top). Typical receiver and system temperatures are shown as $T_{\text {rec }}$ and $T_{\text {sys }}$, respectively. We also show the relevant parameters for previous observations used in this work (bottom). See original references for details.

\begin{tabular}{lccccc}
\hline \hline \multicolumn{5}{c}{ New observations } \\
\hline Line & Freq. (GHz) & Obs. dates & Beam (") & $\eta_{\mathrm{B}}$ & $T_{\text {rec }} / T_{\text {sys }}(\mathrm{K})$ \\
\hline $\mathrm{H}^{12} \mathrm{CO}(3 / 2-1 / 2,2-1)$ & 86.670 & Jun. 00/Aug. 02 & 28 & 0.82 & $70 / 130$ \\
$\mathrm{H}^{13} \mathrm{CO}^{+}(1-0)$ & 86.754 & Jun. 00/Aug. 02 & 28 & 0.82 & $70 / 130$ \\
$\mathrm{SiO}(2-1)$ & 86.847 & Jun. 00/Aug. 02 & 28 & 0.82 & $70 / 130$ \\
$\mathrm{H}^{12} \mathrm{CO}^{+}(1-0)$ & 89.189 & Jan. 01/May 01 & 27 & 0.81 & $60 / 120$ \\
$\mathrm{HO}^{12} \mathrm{C}^{+}(1-0)$ & 89.487 & Jan. 01/May 01 & 27 & 0.81 & $60 / 120$ \\
$\mathrm{SiO}(3-2)$ & 130.269 & Jun. 00/Aug. 02 & 19 & 0.77 & $125 / 225$ \\
$\mathrm{CN}(2-1)$ & 226.875 & Aug. 02 & 11 & 0.58 & $120 / 390$ \\
\hline \multicolumn{5}{c}{ Previous data } \\
\hline \hline $\mathrm{Line}$ & Freq. (GHz) & Telescope & & \\
\hline $\mathrm{HCN}(4-3)$ & 354.505 & JCMT & Tacconi et al. (1994) \\
$\mathrm{CO}(4-3)$ & 461.041 & JCMT & Tacconi et al. (1994) \\
\hline $\mathrm{HCN}(1-0)$ & 89.088 & IRAM PdBI & Tacconi et al. (1994) \\
$\mathrm{CS}(2-1)$ & 97.981 & IRAM PdBI & Tacconi et al. (1997) \\
$\mathrm{CO}(1-0)$ & 115.271 & IRAM PdBI & Schinnerer et al. (2000) \\
$\mathrm{CO}(2-1)$ & 230.538 & IRAM PdBI & Schinnerer et al. (2000) \\
\hline
\end{tabular}

We discuss in Sect. 5 the interpretation of these results in the framework of different chemistry models and summarize the main conclusions of this work in Sect. 6 .

\section{Observations}

The observations have been carried out in four sessions from January 2000 to August 2002 with the IRAM 30 m radiotelescope at Pico Veleta (Spain). We used 3 SIS receivers tuned in single-sideband mode in the $1 \mathrm{~mm}, 2 \mathrm{~mm}$ and $3 \mathrm{~mm}$ bands to observe several transitions of the molecular species shown in Table 1, which summarizes the relevant parameters of these observations. We have obtained single-pointed spectra toward the nucleus of NGC 1068 for all the molecules with the exception of $\mathrm{SiO}, \mathrm{HCO}$ and $\mathrm{H}^{13} \mathrm{CO}^{+}$, for which we obtained partial maps by observing three additional positions on the starburst ring (see Sect. 3.1). The line temperature scale used by default throughout the paper is $T_{\mathrm{MB}}$, i.e., main brightness temperature. $T_{\mathrm{MB}}$ is related to antenna temperature, $T_{\mathrm{A}}^{*}$, by $T_{\mathrm{A}}^{*}=T_{\mathrm{MB}} \times \eta_{\mathrm{B}}$; the values assumed for $\eta_{\mathrm{B}}$ are listed in Table 1 . When explicitly stated, $T_{\mathrm{MB}}$ temperatures are corrected by a source coupling factor, $f_{\mathrm{S}}{ }^{1}$; this factor accounts for the estimated dilution of the source within the beam. To improve the stability of spectral baselines, the observations have been carried out in beam-switching mode, with an azimuthal switch of $\pm 4^{\prime}$ with a frequency of $0.5 \mathrm{~Hz}$. Only linear polynomials were used in the baseline correction.

In this paper we also use the data from previously published HCN, CS and CO observations of NGC 1068 made with

\footnotetext{
${ }^{1}$ Correction for dilution: $T \rightarrow f_{\mathrm{S}} T$ with $f_{\mathrm{S}}=\Omega_{\text {beam }} / \Omega_{\mathrm{S}}$, where $\Omega_{\text {beam }}$ is the beam area and $\Omega_{\mathrm{S}}$ the area of the emitting region estimated from the $\mathrm{CO}(1-0)$ interferometer map.
}

the IRAM Plateau de Bure Interferometer-PdBI $(\mathrm{HCN}(1-0)$ : Tacconi et al. 1994; CS(2-1): Tacconi et al. 1997; CO(1-0) and $\mathrm{CO}(2-1)$ : Schinnerer et al. 2000). Complementary observations of high $J$ transitions $(J=4-3)$ of $\mathrm{CO}$ and $\mathrm{HCN}$, taken at James Clerk Maxwell Telescope-JCMT (Tacconi et al. 1994), are also included. The main parameters of these observations are listed in Table 1.

Hereafter, we will assume a distance to NGC 1068 of $14.4 \mathrm{Mpc}$ (Bland-Hawthorn et al. 1997). This implies $1^{\prime \prime}=72 \mathrm{pc}$. The assumed heliocentric systemic velocity is $v_{\text {sys }}=1137 \mathrm{~km} \mathrm{~s}^{-1}$ (from NASA/IPAC Extragalactic Database (NED)).

\section{The IRAM 30 m survey of NGC 1068}

\subsection{SiO emission in NGC 1068}

NGC 1068 was originally part of a larger extragalactic survey searching for $\mathrm{SiO}$ emission in starbursts (Usero et al. 2003 in prep.). Different mechanisms have been found thus far to explain the enhancement of $\mathrm{SiO}$ abundances in molecular gas in galaxies: either related to recent star formation (NGC 253: García-Burillo et al. 2000), to the disruption of galaxy disks by large-scale shocks (M 82: García-Burillo et al. 2001) or to the X-ray irradiation of molecular clouds (Milky Way: Martín-Pintado et al. 2000).

We show in Fig. 1 the 4 positions over the NGC 1068 disk where we searched for $\mathrm{SiO}$ emission. To better constrain the physical conditions of the gas, we have observed simultaneously the $J=2-1$ and $J=3-2$ rotational transitions of SiO. $\mathrm{SiO}(2-1)$ emission is detected at every offset, while $\mathrm{SiO}(3-2)$, very prominent in the CND, is detected in 2 out of the 3 positions mapped over the ring. The observing grid was 
Table 2. Parameters of gaussian fits to the $\mathrm{SiO} / \mathrm{H}^{13} \mathrm{CO}^{+} / \mathrm{HCO}$ lines observed in NGC 1068. Errors (in brackets) are 1- $\sigma$. For the non-detection of $\mathrm{SiO}(3-2)$ in the $N$ position we give a 3- $\sigma$ upper limit.

\begin{tabular}{|c|c|c|c|c|c|}
\hline Position & Line & $I\left(\mathrm{~K} \mathrm{~km} \mathrm{~s}^{-1}\right)$ & $T_{\text {peak }}(\mathrm{mK})$ & $v-v_{\text {sys }}\left(\mathrm{km} \mathrm{s}^{-1}\right)$ & $\Delta v\left(\mathrm{~km} \mathrm{~s}^{-1}\right)$ \\
\hline \multirow{3}{*}{$\begin{array}{c}\text { CND } \\
\left(0^{\prime \prime}, 0^{\prime \prime}\right)\end{array}$} & $\mathrm{SiO}(2-1)$ & $0.56(0.05)$ & 2.8 & $-26(10)$ & $189(22)$ \\
\hline & $\mathrm{SiO}(3-2)$ & $0.60(0.06)$ & 3.0 & $-36(10)$ & $190(19)$ \\
\hline & $\mathrm{H}^{13} \mathrm{CO}^{+}(1-0)$ & $0.57(0.07)$ & 2.1 & $9(13)$ & $254(38)$ \\
\hline \multirow{4}{*}{$\begin{array}{c}S \\
\left(0^{\prime \prime},-16^{\prime \prime}\right)\end{array}$} & $\mathrm{SiO}(2-1)$ & $0.32(0.04)$ & 1.5 & $-48(9)$ & $200(13)$ \\
\hline & $\mathrm{SiO}(3-2)$ & $0.17(0.04)$ & 2.2 & $-57(9)$ & $69(18)$ \\
\hline & $\mathrm{H}^{13} \mathrm{CO}^{+}(1-0)$ & $0.27(0.04)$ & 1.3 & $-48(13)$ & $200(13)$ \\
\hline & $\operatorname{HCO}(3 / 2-1 / 2,2-1)$ & $0.11(0.03)$ & 1.1 & $-107(13)$ & $100(13)$ \\
\hline \multirow{4}{*}{$\begin{array}{c}\mathrm{N} \\
\left(0^{\prime \prime},+16^{\prime \prime}\right)\end{array}$} & $\mathrm{SiO}(2-1)$ & $0.39(0.08)$ & 1.4 & $-53(29)$ & $261(52)$ \\
\hline & $\mathrm{SiO}(3-2)$ & $<0.25$ & $\ldots$ & $\ldots$ & 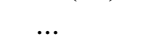 \\
\hline & $\mathrm{H}^{13} \mathrm{CO}^{+}(1-0)$ & $0.44(0.07)$ & 2.8 & $-3(11)$ & $145(27)$ \\
\hline & $\operatorname{HCO}(3 / 2-1 / 2,2-1)$ & $0.20(0.07)$ & 1.4 & $26(23)$ & $138(60)$ \\
\hline \multirow{4}{*}{$\begin{array}{c}\mathrm{E} \\
\left(+16^{\prime \prime}, 0^{\prime \prime}\right)\end{array}$} & $\mathrm{SiO}(2-1)$ & $0.28(0.04)$ & 1.8 & $-60(19)$ & $150(13)$ \\
\hline & $\mathrm{SiO}(3-2)$ & $0.11(0.04)$ & 1.1 & $1(19)$ & $103(36)$ \\
\hline & $\mathrm{H}^{13} \mathrm{CO}^{+}(1-0)$ & $0.21(0.03)$ & 2.2 & $-122(9)$ & $90(13)$ \\
\hline & $\operatorname{HCO}(3 / 2-1 / 2,2-1)$ & $0.18(0.04)$ & 1.6 & $-130(20)$ & $110(13)$ \\
\hline
\end{tabular}

chosen to discriminate between $\mathrm{SiO}$ emission coming from the starburst ring $\left(\mathrm{N}\left[0^{\prime \prime},+16^{\prime \prime}\right], \mathrm{E}\left[+16^{\prime \prime}, 0^{\prime \prime}\right]\right.$ and $\left.\mathrm{S}\left[0^{\prime \prime},-16^{\prime \prime}\right]\right)$ and that coming from the circumnuclear disk $\left(\mathrm{CND}\left[0^{\prime \prime}, 0^{\prime \prime}\right]\right)$. Parameters of the gaussian fits to the lines detected are listed in Table 2.

\subsubsection{Emission in the starburst ring}

These observations show that $\mathrm{SiO}$ emission is widespread in the starburst ring of NGC 1068. Where detected over the ring, $\mathrm{SiO}(3-2)$ lines are narrower than $\mathrm{SiO}(2-1)$ lines. This result can be explained if, contrary to the compactness of $\mathrm{SiO}$ emission in the CND (see below), the emission of $\mathrm{SiO}$ on the ring extends significantly beyond a single $\mathrm{SiO}(3-2)$ beam. Within the errors, the $I(\mathrm{SiO}(3-2)) / I(\mathrm{SiO}(2-1))$ integrated intensity ratios are $\sim 0.5$ in the two positions with detection of the $2 \mathrm{~mm}$ line. These ratios are slightly lowered to $0.4 \pm 0.1$ if we apply a correction due to the different coupling factors of the 3-2 and 2-1 beams with the source (correcting for dilution of the nearly one-dimensional elongated arm inside the beams, i.e., by a factor $\sim 19^{\prime \prime} / 28^{\prime \prime}$ ).

There are two precedents for the detection of largescale $\mathrm{SiO}$ emission associated with ongoing star formation: NGC 253 (García-Burillo et al. 2000) and M 82 (García-Burillo et al. 2001). The derived enhancement of $\mathrm{SiO}$ abundances $\left(X(\mathrm{SiO}) \sim\right.$ a few $\left.10^{-10}-10^{-9}\right)$ takes place on scales of several hundred $\mathrm{pc}$ in these starbursts and has been interpreted as a signature of shocks driven by YSO, SN explosions and/or density waves. In the starburst ring of NGC 1068, a significant fraction of the stellar population ( $\sim 40 \%$ of the total optical light; González-Delgado et al. 2001) has typical ages $\leq 10^{7} \mathrm{yr}$. This supports that a recent short burst of star formation has occurred coevally throughout the ring on a time-scale of $\sim 10^{6} \mathrm{yr}$ (Davies et al. 1998).

Beside the detection of the $1-0$ line of $\mathrm{H}^{13} \mathrm{CO}^{+}$(see Fig. 1), which is $93 \mathrm{MHz}$ redshifted with respect to the $\mathrm{SiO}(2-1)$ line, we have detected the emission of the strongest hyperfine component $(F=2-1)$ of the $J=3 / 2-1 / 2$ line of $\mathrm{HCO}$ over the starburst ring. Observations of $\mathrm{HCO}$ in galactic clouds suggest that the abundance of this molecule is enhanced in Photon Dominated Regions (PDR). More recently, García-Burillo et al. (2002) have reported on the detection of widespread HCO emission in the nuclear starburst of M 82, where it traces the propagation of PDR chemistry in the disk. Based on studies of HCO emission in Galactic PDR (Schenewerk et al. 1986, 1988), it is plausible to suppose that the HCO lines should be optically thin also in the starburst ring of $\mathrm{NGC} 1068$. For $\mathrm{H}^{13} \mathrm{CO}^{+}$we also consider optically thin emission and the same excitation temperature as that assumed for HCO. These are reasonable guesses, especially for $T_{\mathrm{ex}}$, as the two molecules have similar critical densities for the examined transitions. In this case, the calculation of the HCOto- $\mathrm{H}^{13} \mathrm{CO}^{+}$column density ratio is straightforward using the expression (Schenewerk et al. 1988):

$\frac{N(\mathrm{HCO})}{N\left(\mathrm{H}^{13} \mathrm{CO}^{+}\right)} \simeq \frac{12}{5} \frac{I_{\mathrm{HCO}} A_{\mathrm{HCO}}^{-1}}{I_{\mathrm{H}^{13} \mathrm{CO}^{+}} A_{\mathrm{H}^{13} \mathrm{CO}^{+}}^{-1}}$

where $N$ is the total column density, $I$ is the integrated intensity, and $A$ is the Einstein coefficient of the transition. We infer an average value for $N(\mathrm{HCO}) / N\left(\mathrm{H}^{13} \mathrm{CO}^{+}\right)$ of $\sim 8$. Adopting an average fractional abundance for $\mathrm{H}^{13} \mathrm{CO}^{+}$ of $10^{-10}$ (García-Burillo et al. 2000, 2001), we derive $X(\mathrm{HCO}) \sim 8 \times 10^{-10}$. The estimated $N(\mathrm{HCO}) /\left(\mathrm{H}^{13} \mathrm{CO}^{+}\right)$abundance ratios in prototypical PDR range from 30 , in the Orion Bar, to 3, in NGC 7023 (Schilke et al. 2001).

Altogether, the detection of widespread $\mathrm{SiO}$ and HCO emission in the starburst ring of NGC 1068 can be naturally explained by the chemical processing of molecular gas after a recent episode of star formation. 


\subsection{2. $\mathrm{SiO}$ emission in the circumnuclear disk (CND)}

As is shown in Fig. 1, the spatial resolution of the $30 \mathrm{~m}$ in the 3-2 line (19") guarantees that the $\mathrm{SiO}(3-2)$ emission detected toward the CND has little if any contamination from the starburst ring (of $\sim 30^{\prime \prime}$ diameter). The similar line-widths of the $2-1$ and $3-2 \mathrm{SiO}$ spectra at $\left(0^{\prime \prime}, 0^{\prime \prime}\right)$ provide further evidence that the bulk of the central $\mathrm{SiO}(3-2)$ emission comes from the CND. Furthermore, the linewidth of both $\mathrm{SiO}$ lines $\left(F W Z P=350 \mathrm{~km} \mathrm{~s}^{-1}\right)$ coincides with the total line width of the $\mathrm{CO}(1-0)$ emission integrated within the $\mathrm{CND}$, as derived from the interferometer map of Schinnerer et al. (2000). While the $\mathrm{SiO}(3-2)$ line at $\left(0^{\prime \prime}, 0^{\prime \prime}\right)$ has no significant contribution from the starburst ring, the situation is less clear in the case of the $\mathrm{SiO}(2-1)$ spectrum: the $28^{\prime \prime} 30 \mathrm{~m}$ beam at half power may pick up emission coming mostly from the southern ridge of the star forming ring (see Fig. 1). Taking into account that the $\mathrm{SiO}(2-1)$ line temperatures measured over the ring are a factor of 2 lower than in the CND, the derived upper limit for the "alien" contribution to the $\mathrm{SiO}(2-1) \mathrm{CND}$ spectrum is $\sim 25 \%$, at most.

The $I(\mathrm{SiO}(3-2)) / I(\mathrm{SiO}(2-1))$ ratio in the CND is of $0.7 \pm 0.1$, once corrected for the contribution of the starburst ring to the $2-1 \mathrm{CND}$ line $(\times 1 / 0.75)$ and for the two-dimensional beam dilution of the CND $\left(\times\left(28^{\prime \prime} / 19^{\prime \prime}\right)^{2}\right)$. Simultaneously, we have evaluated the contribution of the CND to the $\mathrm{SiO}(2-1)$ spectra in the ring to be, at most, $\sim 25 \%$. When we correct for this effect, the $I(\mathrm{SiO}(3-2)) / I(\mathrm{SiO}(2-1))$ average ratio on the ring derived in Sect. 3.1.1 is raised to $0.5 \pm 0.1$, i.e., a factor 1.5 smaller than the ratio in the CND. Although the difference is only marginal, it suggests that the excitation of $\mathrm{SiO}$ lines in the CND is different from that of the ring. In particular gas densities in the CND could be larger by a factor of $\sim 4$ compared to the starburst ring.

A relevant contribution from the molecular bar to the $\mathrm{SiO}$ emission detected at $\left(0^{\prime \prime}, 0^{\prime \prime}\right)$ is also very unlikely for several reasons. First, the bar hardly stands out in the HCN and CS interferometer maps of NCG 1068 (Tacconi et al. 1997): this is a relevant result, as the critical densities of $\mathrm{HCN}(1-0)$ and $\mathrm{CS}(2-1)$ lines are similar to that of $\mathrm{SiO}(2-1)$. Second, while weak $\mathrm{CO}$ emission is detected along the bar, it is significant only at $v<v_{\text {sys }}$ : this is at odds with the observed $\mathrm{SiO}$ line profiles, roughly symmetric on both sides around $v_{\text {sys. }}$.

Most remarkably, there is no evidence for significant recent star formation in the CND itself. Several multiwavelength criteria have classified the nucleus of NGC 1068 as a pure Seyfert nucleus, with little contribution from a nuclear starburst (MIR: Laurent et al. 2000; NIR: Imanishi 2002; Optical/Near-UV: Cid-Fernandes et al. 2001); the compact starburst emits $\leq 1 \%$ of the total IR luminosity (Marco \& Brooks 2003). The circumnuclear stellar population is concentrated in a $50 \mathrm{pc}$ core of "post-starburst" intermediate age stars (age $\geq 5-16 \times 10^{8} \mathrm{yr}$ ) (Thatte et al. 1997).

We can exclude star formation either inside or outside the $\mathrm{CND}$ as the mechanism explaining the emission of $\mathrm{SiO}$ detected at $\left(0^{\prime \prime}, 0^{\prime \prime}\right)$. This poses the problem of the origin of $\mathrm{SiO}$ emission in the CND. The energy budget inside the CND seems to be largely dominated by the AGN itself; thus the chemistry of molecular gas, in particular the silicon chemistry, could be driven by non-stellar processes. We discuss in Sect. 5.1 how the high abundances derived for $\mathrm{SiO}$ in the CND might be linked to the onset of XDR chemistry.

\subsection{Emission of reactive ions in NGC 1068: The $\mathrm{HOC}^{+} / \mathrm{HCO}^{+}$isomers}

Detailed chemical models of XDR predict enhanced abundances of some reactive ions (e.g., $\mathrm{H}_{3}^{+}, \mathrm{HCO}^{+}, \mathrm{SO}^{+}, \mathrm{CO}^{+}$and $\mathrm{HCNH}^{+}$) as well as related neutral species (such as $\mathrm{CN}$ and HCN) (Maloney et al. 1996; Black 1998a,b; Lepp \& Dalgarno 1996). The tentative detection of $\mathrm{CO}^{+}$in the radio galaxy Cygnus A (Fuente et al. 2000) suggests that reactive ions may be used as an efficient diagnostic tool to study XDR chemistry in AGN. As part of this multi-species survey of NGC 1068, we have observed the $1-0$ line of $\mathrm{HCO}^{+}$toward the CND. Most importantly, we have also searched for emission of its metastable isomer, $\mathrm{HOC}^{+}$. There is recent observational evidence that $X\left(\mathrm{HCO}^{+}\right) / X\left(\mathrm{HOC}^{+}\right)$ratios, usually ranging from 300-6000 for dense molecular clouds in our Galaxy (Apponi et al. 1997, 1999), can reach values as low as 50-100 in UV-irradiated clouds (e.g., the prototypical PDR NGC 7023: Fuente et al. 2003). These results urged us to estimate the $\mathrm{HCO}^{+}$-to- $\mathrm{HOC}^{+}$ ratio in the X-ray bathed environment of an AGN.

Figure 2 shows the $J=1-030 \mathrm{~m}$ spectra of $\mathrm{HCO}^{+}$and $\mathrm{HOC}^{+}$observed toward the CND of NGC 1068. The emission of both species is detected. The interferometer $\mathrm{HCO}^{+}$map of Kohno et al. (2001) shows that the CND largely dominates the emission of $\mathrm{HCO}^{+}$in the inner $3 \mathrm{kpc}$ of NGC 1068. Moreover, we can estimate a conservative upper limit for the contribution of the starburst ring to the $\mathrm{HCO}^{+}$emission detected at $\left(0^{\prime \prime}, 0^{\prime \prime}\right)$. Following the same procedure used in Sect. 3.1.2, here adapted to $\mathrm{H}^{13} \mathrm{CO}^{+}$, we estimate that $<30 \%$ of the $\left(0^{\prime \prime}, 0^{\prime \prime}\right) \mathrm{H}^{13} \mathrm{CO}^{+}$ emission can be attributed to the starburst ring. We can reasonably extrapolate this estimate to $\mathrm{HCO}^{+}$. Additional evidence, similar to the one discussed in Sect. 3.1.2, supports that the $30 \mathrm{~m} \mathrm{HCO}^{+}$spectrum is heavily dominated by emission coming from the CND.

The most remarkable result is the tentative detection of the $\operatorname{HOC}^{+}(1-0)$ line, the first thus far obtained in an external galaxy. $\mathrm{HOC}^{+}(1-0)$ emission is detected over $2 \sigma$ levels in a $215 \mathrm{~km} \mathrm{~s}^{-1}$ velocity range $\left(\sim\left[-65 \mathrm{~km} \mathrm{~s}^{-1},+150 \mathrm{~km} \mathrm{~s}^{-1}\right]\right)$. The emission integrated within this velocity window reaches a $8.5 \sigma$ significance level. The line profile of $\mathrm{HOC}^{+}$is noticeably asymmetrical with respect to $v_{\text {sys }}$ : $\mathrm{HOC}^{+}$emission is mostly detected at red velocities. As is shown in Fig. 2, $\mathrm{HCO}^{+}$-to$\mathrm{HOC}^{+}$intensity ratios for $v \geq v_{\text {sys }}$ range from $\sim 40$ to 100 . These surprisingly low values rival the lowest values thus far derived in $\mathrm{PDR}$. The low $\mathrm{HCO}^{+}$-to- $\mathrm{HOC}^{+}$intensity ratio measured in the CND of NGC 1068 suggests that the chemistry of molecular gas could be driven by the pervading X/UV irradiation coming from the Seyfert 2 nucleus. Most remarkably, the asymmetry of the $\mathrm{HOC}^{+}$line profile suggests that whatever causes the enhancement of this active ion, the process responsible seems to be unevenly efficient inside the CND. As it is discussed in Sect. 5.2, X-ray driven chemistry in the CND may 


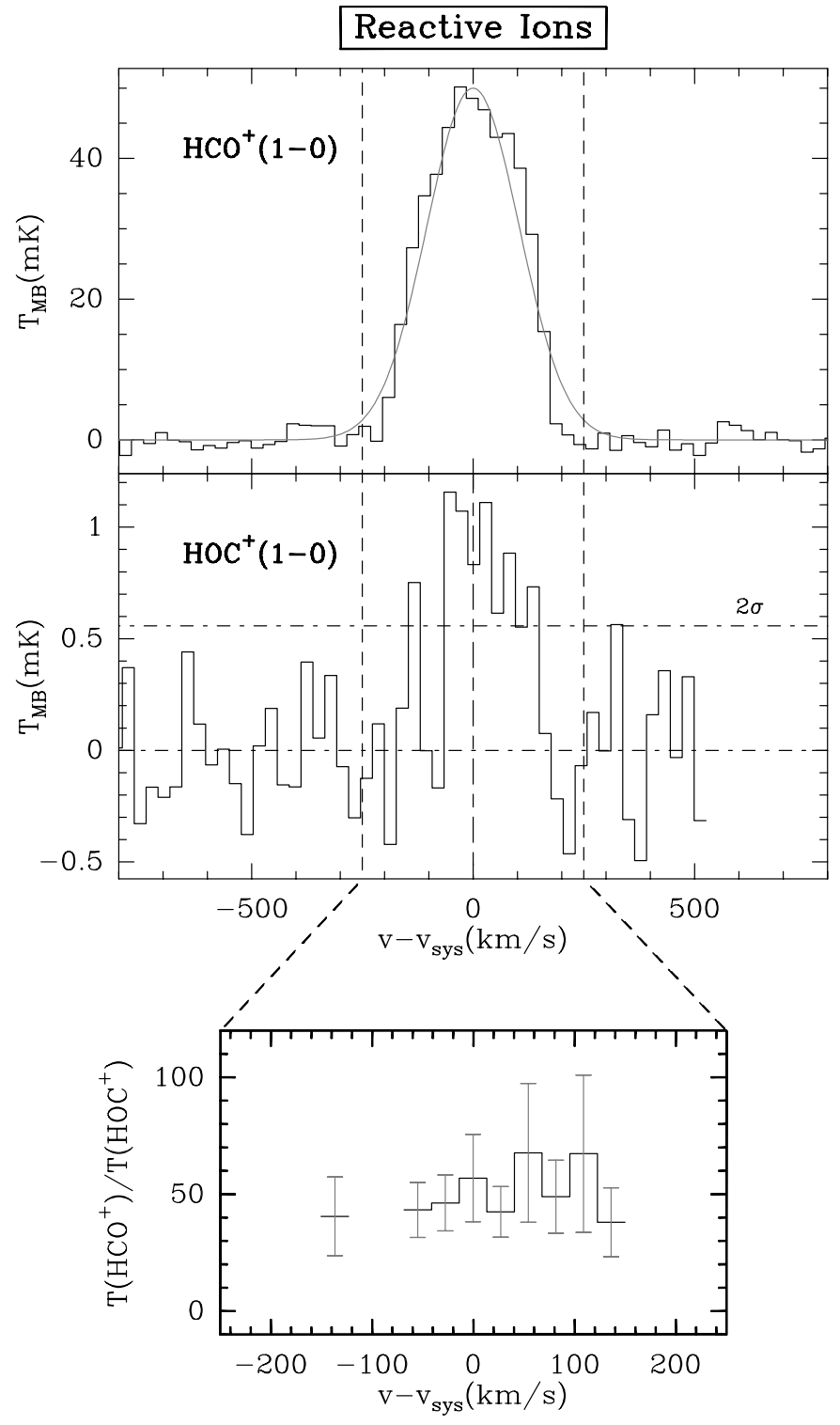

Fig. 2. Top and middle: $\mathrm{HCO}^{+}(1-0)$ and $\mathrm{HOC}^{+}(1-0)$ spectra of the CND of NGC 1068. Bottom: $\mathrm{HCO}^{+}(1-0)$-to- $\mathrm{HOC}^{+}(1-0)$ temperature ratio profile derived for channels fulfilling $T\left[\mathrm{HOC}^{+}(1-0)\right]>2 \sigma$. Error bars are $\pm \sigma$.

satisfactorily explain a dramatic change in the $\mathrm{HCO}^{+}$-to- $\mathrm{HOC}^{+}$ abundance ratio.

\section{3. $C N$ emission in NGC 1068}

$\mathrm{CN}$ is a high-dipole radical typically found in dense regions $\left(\sim 10^{5} \mathrm{~cm}^{-3}\right)$. The abundance of $\mathrm{CN}$ is strongly linked to that of HCN. Theoretical models (Lepp \& Dalgarno 1996) predict large $\mathrm{CN}$-to-HCN abundance ratios $(>1)$ in XDR. The comparison of the $\mathrm{CN}$ and $\mathrm{HCN}$ emission may thus provide a suitable diagnostic of the relevance of X-rays in the chemistry of the CND.

The $\mathrm{CN}(2-1)$ transition is split up into 18 hyperfine lines that appear blended into three groups at frequencies $\sim 226.9 \mathrm{GHz}, \sim 226.7 \mathrm{GHz}$ and $\sim 226.4 \mathrm{GHz}$. We were able to observe the two most intense groups of the transition (the $226.9 \mathrm{GHz}$ and $226.7 \mathrm{GHz}$ groups, hereafter referred to as high frequency and low frequency respectively), although the low frequency group was only partially covered by the spectral bandwidth. The beam size at this frequency $\left(11^{\prime \prime}\right)$ guarantees that the detected $\mathrm{CN}(2-1)$ emission must be coming from the CND.

The $\mathrm{CN}(2-1)$ spectrum is shown in Fig. 4 (main-beam temperature scaled to the CND; see Sect. 4.2). The measured highfrequency-to-low-frequency intensity ratio is below the expected value for the optically thin limit (high/low $\sim 1.64 \pm 0.14$ instead of 1.80). However, this estimate is hampered by the insufficient baseline coverage in the spectrum.

\section{Molecular gas inventory of the CND}

Understanding the peculiar chemistry of molecular gas revealed in the CND of NGC 1068 requires a global analysis of its molecular inventory. Furthermore, higher spatial resolution is key to extracting the maximum information from the $30 \mathrm{~m}$ spectra of $\mathrm{SiO}, \mathrm{HCO}^{+}$and $\mathrm{HOC}^{+}$discussed above.

With this aim we have included in our analysis the information provided by published interferometer maps of NGC 1068 obtained for CO, CS and HCN (Schinnerer et al. 2000; Tacconi et al. 1994, 1997). These maps can help to improve our knowledge on the molecular abundances for species such as HCN or $\mathrm{CS}$ in the CND; due to their high spatial resolution, these observations are not hampered by source confusion between the CND itself and the starburst ring. In particular, the CO interferometer map allows us to estimate the molecular hydrogen column densities in the CND. Moreover, the spatio-kinematical information of the $\mathrm{CO}$ interferometer map is used for calculating the size and the location inside the CND of the gas components emitting at different velocities. Altogether, this information is employed in Sect. 4.3 to estimate via LVG models the abundances of several molecular species in the CND, separately, for the relevant velocity components.

\subsection{Morphology of the CND: The interferometer $\mathrm{CO}(1-0)$ map}

Figure 3 represents the $\mathrm{CO}(1-0)$ spatially integrated spectrum of the CND of NGC 1068. The line emission profile has been obtained by integrating the $\mathrm{CO}(1-0)$ interferometer data of Schinnerer et al. (2000) inside a $6^{\prime \prime} \times 4^{\prime \prime}$-rectangular region which contains the bulk of the $\mathrm{CO}$ emission in the CND. According to Schinnerer et al. (2000)'s estimates, we expect little zero-spacing flux missing in the CND integrated spectrum/map. Molecular gas in the CND is not evenly distributed around the AGN: two conspicuous knots (denoted as E[1", $\left.0^{\prime \prime}\right]$ and $\mathrm{W}\left[-1.5^{\prime \prime}, 0^{\prime \prime}\right]$ knots) form an asymmetrical ring around the AGN (see Fig. 1 of Schinnerer et al. 2000 and Fig. 1 in this work). The asymmetrical distribution of molecular gas in the CND is reflected by the profile of Fig. 3: the $\mathrm{CO}(1-0)$ emission integrated for $v<v_{\text {sys }}$ (hereafter, called blue component) is $\sim 2$ times that measured for $v>v_{\text {sys }}$ (hereafter, called red component). As expected for a disk rotating around the AGN, 


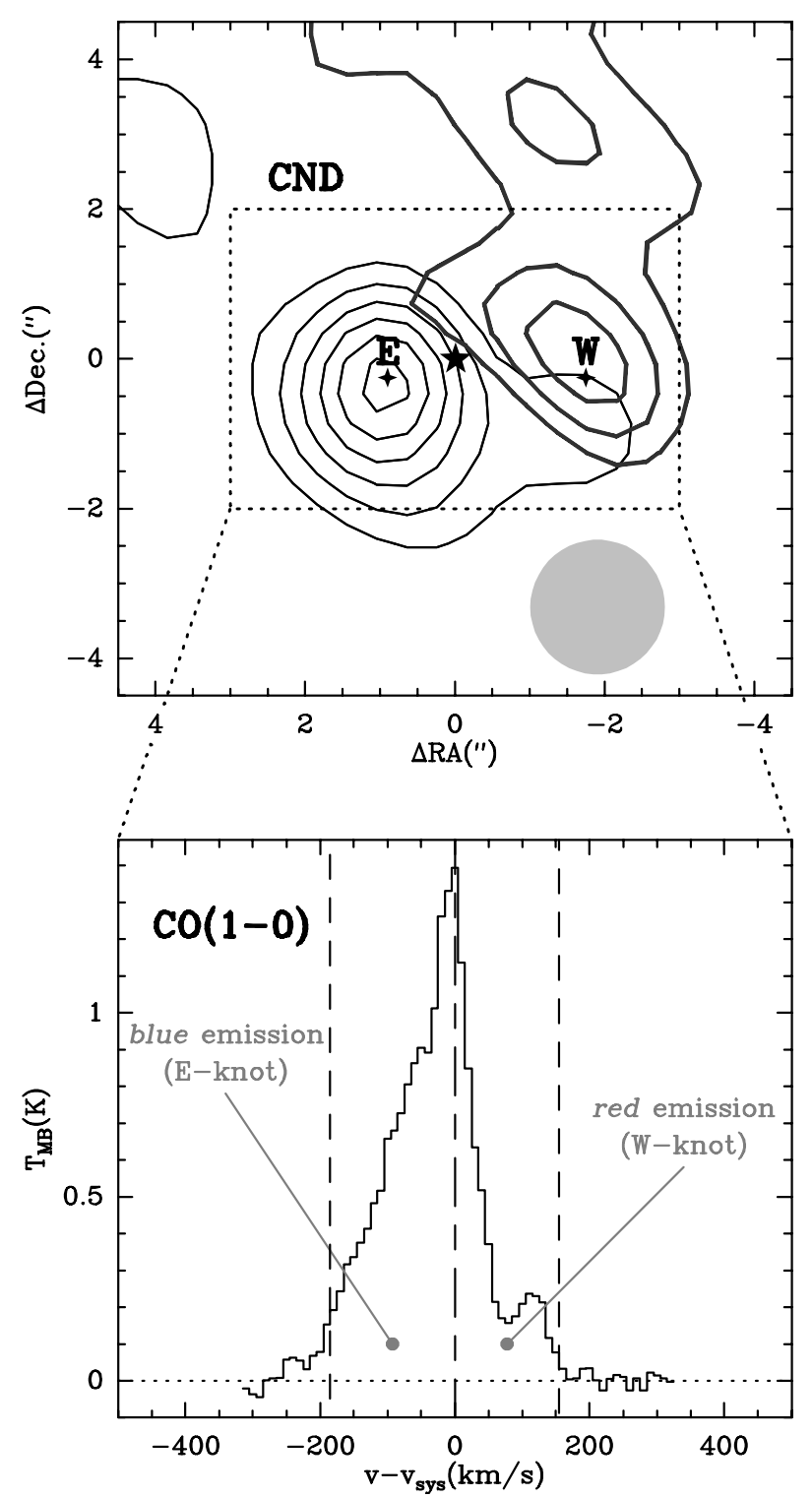

Fig. 3. Top panel: integrated intensity maps of $\mathrm{CO}(1-0)$ toward the CND of NGC 1068 obtained for the blue (thin contours: from $6 \sigma$ by steps of $3 \sigma ; \sigma=0.67 \mathrm{~K} \mathrm{~km} \mathrm{~s}^{-1}$ ) and red (thick contours: same levels with $\sigma=0.47 \mathrm{~K} \mathrm{~km} \mathrm{~s}^{-1}$ ) emission components as defined in text (see also bottom panel). The maps have been derived from the data of Schinnerer et al. (2000). The starred marker highlights the AGN locus. Bottom panel: integrated spectrum of $\mathrm{CO}(1-0)$ emission in the CND. The $\mathrm{W}$ and $\mathrm{E}$ knots in the CO map correspond, respectively, to the red and blue components in the spectrum.

the emission coming from the $\mathrm{E}$ and $\mathrm{W}$ knots roughly correspond, respectively, to the blue and red components defined above. This is illustrated in Fig. 3.

The full sizes of the $\mathrm{E}$ and $\mathrm{W}$ knots, deconvolved by the $1.8^{\prime \prime} \times 1.8^{\prime \prime}$ beam, are alike: $F W Z P \simeq 2.2^{\prime \prime}$. Therefore we deduce similar areas for the blue and red emitting regions: $\Omega_{\text {source }} \simeq \pi \times 1.1^{2} \operatorname{arcsec}^{2}=3.8 \operatorname{arcsec}^{2}$.

\subsection{Molecular line profiles of the CND}

Figure 4 displays all the molecular lines observed in this work toward the CND of NGC 1068. This includes the $30 \mathrm{~m}$
Table 3. Integrated intensities of the spectra of Fig. 4 in the blue (Col. 2) and red (Col. 3) components. Column 4 = blue-to-red (eastto-west) ratio of mean temperatures. Errors (in brackets) are 1- $\sigma$.

\begin{tabular}{lrrr}
\hline \hline Transition & $I_{\text {blue }}\left(\mathrm{K} \mathrm{km} \mathrm{s}^{-1}\right)$ & $I_{\text {red }}\left(\mathrm{K} \mathrm{km} \mathrm{s}^{-1}\right)$ & $R_{\mathrm{E} / \mathrm{W}}$ \\
\hline $\mathrm{CO}(1-0)$ & $127.1(0.9)$ & $60.1(0.9)$ & $1.77(0.03)$ \\
$\mathrm{CO}(2-1)$ & $95.5(0.4)$ & $51.2(0.3)$ & $1.56(0.01)$ \\
\hline $\mathrm{CS}(2-1)$ & $8.7(0.6)$ & $3.2(0.5)$ & $2.24(0.40)$ \\
\hline $\mathrm{HCN}(1-0)$ & $121.5(1.7)$ & $91.1(1.5)$ & $1.12(0.02)$ \\
\hline $\mathrm{SiO}(2-1)$ & $8.5(0.8)$ & $6.2(0.7)$ & $1.15(0.18)$ \\
$\mathrm{SiO}(3-2)$ & $4.7(0.5)$ & $2.5(0.4)$ & $1.55(0.32)$ \\
\hline $\mathrm{H}^{13} \mathrm{CO}^{+}(1-0)$ & $6.3(0.8)$ & $6.9(0.7)$ & $0.77(0.13)$ \\
$\mathrm{HCO}^{+}(1-0)$ & $167.2(2.2)$ & $158.4(2.1)$ & $0.88(0.02)$ \\
$\mathrm{HOC}^{+}(1-0)$ & $2.1(0.5)$ & $2.8(0.4)$ & $0.61(0.17)$ \\
\hline
\end{tabular}

spectra of $\mathrm{SiO}, \mathrm{HOC}^{+}, \mathrm{HCO}^{+}, \mathrm{H}^{13} \mathrm{CO}^{+}$and $\mathrm{CN}$ (panels 4-9 in Fig. 4). Temperatures have been rescaled assuming that the emission comes from the $6^{\prime \prime} \times 4^{\prime \prime}$-rectangular region containing the CND. Furthermore, we represent in panels 1-3 of Fig. 4, the CND spectra of CO, CS and HCN obtained from published interferometer maps. Similarly to CO (see Sect. 4.1), these CND spectra have been obtained by integrating the $\mathrm{HCN}$ and CS emission inside the $6^{\prime \prime} \times 4^{\prime \prime}$ rectangular region which contains the bulk of the CND flux in both interferometer maps. We can redefine more precisely what we call red and blue velocities, ascribed, as argued above, to the $\mathrm{W}$ and $\mathrm{E}$ knots, respectively: based on the observed molecular profiles of Fig. 4, most of the molecular emission detected at red (blue) velocities for all species arises within the interval $0<v-v_{\text {sys }}<155 \mathrm{~km} \mathrm{~s}^{-1}$ $\left(-185 \mathrm{~km} \mathrm{~s}^{-1}<v-v_{\mathrm{sys}}<0\right)$. Integrated intensities in the blue and red components of the spectra are listed in Table 3 .

There are noticeable differences between the line shapes of the CND spectra shown in Fig. 4. We find line profiles dominated by emission at blue velocities for $\mathrm{CO}$ and $\mathrm{CS}$, while line profiles of $\mathrm{HCO}^{+}, \mathrm{H}^{13} \mathrm{CO}^{+}$and $\mathrm{HOC}^{+}$are dominated by red emission. As argued in Sect. 3.2, $\mathrm{HOC}^{+}$represents an extreme case as the bulk of the $\mathrm{HOC}^{+}$emission is detected at red velocities. $\mathrm{HCN}$ profiles are rather symmetrical with respect to $v_{\text {sys }}$. Finally, the $\mathrm{SiO}$ line profiles represent a case somewhat intermediate between $\mathrm{HCN}$ and $\mathrm{CO}$. These differences are quantified in Fig. 4 and Table 3, which show the blue-to-red (eastto-west $)$ average brightness temperature ratio $\left(R_{\mathrm{E} / \mathrm{w}}\right)$ for all the CND spectra (except for $\mathrm{CN}(2-1)$, for which the determination of the blue and red components is hampered by the partial blending of the lines). $R_{\mathrm{E} / \mathrm{w}}$ ranges from $2.2 \pm 0.4(\mathrm{CS}(2-1))$ to $0.6 \pm 0.2\left(\mathrm{HOC}^{+}(1-0)\right)$, i.e., from one extreme to the other, this ratio changes by a significant factor $(\sim 4)$ among the observed molecules. Figure 5 also illustrates this result: the $\mathrm{HCN}(1-$ $0) / \mathrm{CO}(1-0)$ temperature ratio is a factor of 2-3 larger for the red component than for the blue component. Furthermore, the $\mathrm{CO}(2-1) / \mathrm{CO}(1-0)$ ratio profile, shown in Fig. 5, is also 


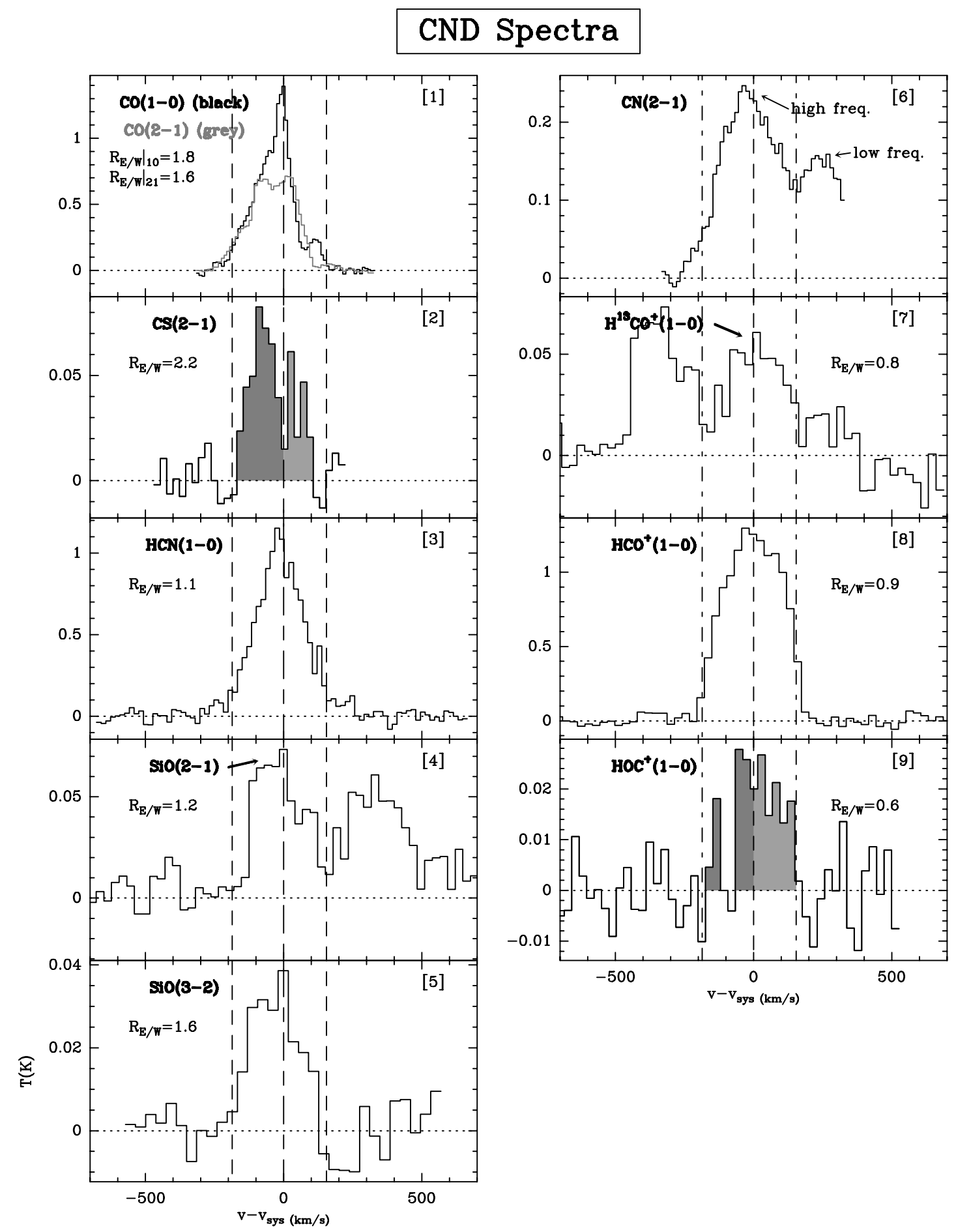

Fig. 4. Molecular lines in the CND. Sub-panels are labeled with the name of the line displayed. Sub-panels 1 to 3 are derived from interferometer data (Tacconi et al. 1994, 1997; Schinnerer et al. 2000); panels 4 to 9 show single-dish spectra observed towards the nucleus (temperatures corrected by dilution effects assuming that the emission is coming from the CND). Two vertical point-dashed lines at $v-v_{\text {sys }}=-185 \mathrm{~km} \mathrm{~s}{ }^{-1}$ and $155 \mathrm{~km} \mathrm{~s}^{-1}$, delimit the blue and red kinematical components. For each line, the blue-to-red (east-to-west) average brightness temperature ratio $\left(R_{\mathrm{E} / \mathrm{W}}\right)$ is indicated.

asymmetrical with respect to $v_{\text {sys }}$ : the $(2-1)$-to-(1-0) ratio reaches higher-than-one values within a $70 \mathrm{~km} \mathrm{~s}^{-1}$ interval at red velocities, while it oscillates between 0.6 and 0.8 for the blue component.

Taken together, these results suggest that there is a chemical differentiation between the $\mathrm{E}$ and $\mathrm{W}$ knots of the CND.

\subsection{Molecular gas abundances in the CND}

\subsubsection{LVG models}

We have used single-component Large Velocity Gradient (LVG) models to estimate the column densities of the observed molecular species under certain assumptions which are the 

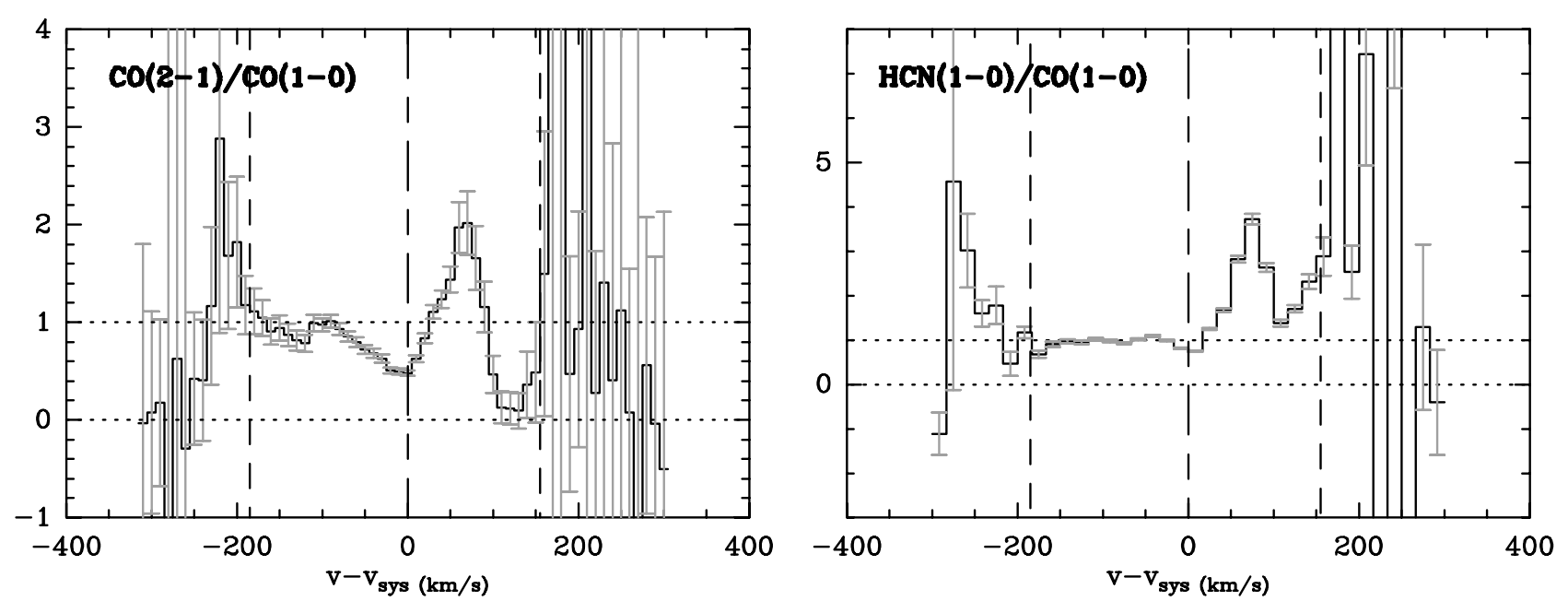

Fig. 5. Temperature ratio profiles derived from spectra of Table 4. The left panel shows the $\mathrm{CO}(2-1)$-to- $\mathrm{CO}(1-0)$ ratio, and the right panel the $\mathrm{HCN}(1-0)$-to- $\mathrm{CO}(1-0)$ ratio. Error bars are $\pm 3 \sigma$.

basis of all our calculations. First, we assume that the kinetic temperature $\left(T_{\mathrm{K}}\right)$ of molecular gas in the CND is $50 \mathrm{~K}$. This value was derived by Sternberg et al. (1994) from the LVG analysis of several CO emission-lines observed toward the CND. Therefore this value can be taken as a conservative lower limit for $T_{\mathrm{K}}$. Furthermore, we adopt in our calculations an isotopic ratio of ${ }^{12} \mathrm{C} /{ }^{13} \mathrm{C}=40$ (Wannier 1980).

It has been previously reported that LVG models of $\mathrm{CO}$ emission in PDR-type environments can lead to inconsistencies related to spatial fine structure, density and kinetic temperature (see the case of M 82 in Mao et al. 2000). However, high $J$-number transitions (out to $\operatorname{CO}(J=7-6)$ ), not available for NGC 1068, are required to constrain LVG-parameters sufficiently to search for inconsistencies.

As argued in Sect. 4.1, the interferometric CO maps reveal two distinct knots (E-W) in the CND. These knots have similar sizes $\left(\Omega_{\text {source }} \sim 3.8 \operatorname{arcsec}^{2}\right)$ and can be identified with two adjacent velocity components of emission in the spectra. As discussed in Sect. 4.2, the relative intensity ratio between these components depends on the molecular species. In our calculations we thus give our estimates of abundance ratios separately for the E/blue and $\mathrm{W} /$ red components. All source brightness temperatures $\left(T_{\mathrm{S}}(\mathrm{E} / \mathrm{W})\right)$, listed in Table 4 , have been derived from the CND temperature scale used in Fig. 4, corrected by a dilution factor $f=\Omega_{\mathrm{CND}} / \Omega_{\text {source }}$.

The range of LVG solutions $\left(n\left(\mathrm{H}_{2}\right), N / \Delta v\right)$ are determined straightforwardly for $\mathrm{SiO}, \mathrm{CO}, \mathrm{HCN}$ and $\mathrm{CN}$ from the observed line ratios and the source brightness temperatures. In the case of $\mathrm{SiO}$, we fit the (3-2)-to-(2-1) ratio and the $2-1$ line source temperature. Correction for contamination from the ring is taken into account for $\mathrm{SiO}(2-1)$ (also for $\mathrm{HCO}^{+}(1-0)$; see below). For $\mathrm{CO}$ and $\mathrm{HCN}$ we use the (4-3)-to-(1-0) line ratios and the 1-0 line source temperatures; $4-3$ line temperatures of $\mathrm{CO}$ and $\mathrm{HCN}$ are derived from single-dish data published by Tacconi et al. (1994). In the case of CN, we fit the ratio of the two fine structure lines and the low-frequency line source temperature. However, and due to partial blending of the two fine groups, LVG solutions refer to global
Table 4. Mean temperatures in the East and West knots of the CND after correction for dilution: Col. 1 = name of the line; Col. $2=$ mean temperature in the East-knot; Col. 3 = idem in the West-knot.

\begin{tabular}{lcc}
\hline \hline Transition & $\langle T\rangle_{\mathrm{E}}(\mathrm{K})$ & $\langle T\rangle_{\mathrm{W}}(\mathrm{K})$ \\
\hline $\mathrm{CO}(1-0)$ & 4.34 & 2.44 \\
$\mathrm{CO}(2-1)$ & 3.26 & 2.08 \\
$\mathrm{CO}(4-3)$ & 7.58 & 6.50 \\
\hline $\mathrm{HCN}(1-0)$ & 4.15 & 3.71 \\
$\mathrm{HCN}(4-3)$ & 1.24 & 0.62 \\
\hline $\mathrm{SiO}(2-1)$ & 0.29 & 0.25 \\
$\mathrm{SiO}(3-2)$ & 0.16 & 0.10 \\
\hline & \multicolumn{2}{c}{$T_{\text {peak }}(\mathrm{K})$} \\
$\mathrm{CN}(2-1$, high freq. $)$ & \multicolumn{2}{c}{0.51} \\
$\mathrm{CN}(2-1$, low freq. $)$ & \multicolumn{2}{c}{0.} \\
\hline $\mathrm{HCO}^{+}(1-0)$ & 5.73 & 6.50 \\
$\mathrm{H}^{13} \mathrm{CO}^{+}(1-0)$ & 0.21 & 0.28 \\
$\mathrm{HOC}(1-0)$ & 0.07 & 0.12 \\
\hline $\mathrm{CS}^{+}(2-1)$ & 0.30 & 0.13 \\
\hline
\end{tabular}

abundances with no distinction between red and blue velocity components. The LVG solution for $\mathrm{H}^{12} \mathrm{CO}^{+}$is obtained by fitting both the $\mathrm{H}^{12} \mathrm{CO}^{+}$-to- $\mathrm{H}^{13} \mathrm{CO}^{+}$temperature ratio measured for the 1-0 line and the $\mathrm{H}^{12} \mathrm{CO}^{+}(1-0)$ source temperature. We have implicitly assumed that the derived density solution $n\left(\mathrm{H}_{2}\right)$ can be considered as common for both $\mathrm{H}^{12} \mathrm{CO}^{+}$and $\mathrm{H}^{13} \mathrm{CO}^{+}$. In the case of $\mathrm{HOC}^{+}, \mathrm{LVG}$ estimates are only obtained in the $\mathrm{W}$ knot, since the signal-to-noise ratio of the integrated emission at blue velocities (E knot) is too low $(<5)$. The estimate of $N / \Delta v$ for $\mathrm{HOC}^{+}$, only observed in the 1-0 line, rests on the assumption of a value for $n\left(\mathrm{H}_{2}\right)$, here taken from $\mathrm{H}^{12} \mathrm{CO}^{+}$. This approach is justified as $\mathrm{HOC}^{+}$and $\mathrm{HCO}^{+}$are known to be formed/destroyed in chemical reactions taking place in the same gas clouds. Similarly, $N / \Delta v$ values for CS are derived assuming for this molecule the same gas density inferred from $\mathrm{HCN}$ in order to fit the $\mathrm{CS}(2-1)$ source brightness temperature. 

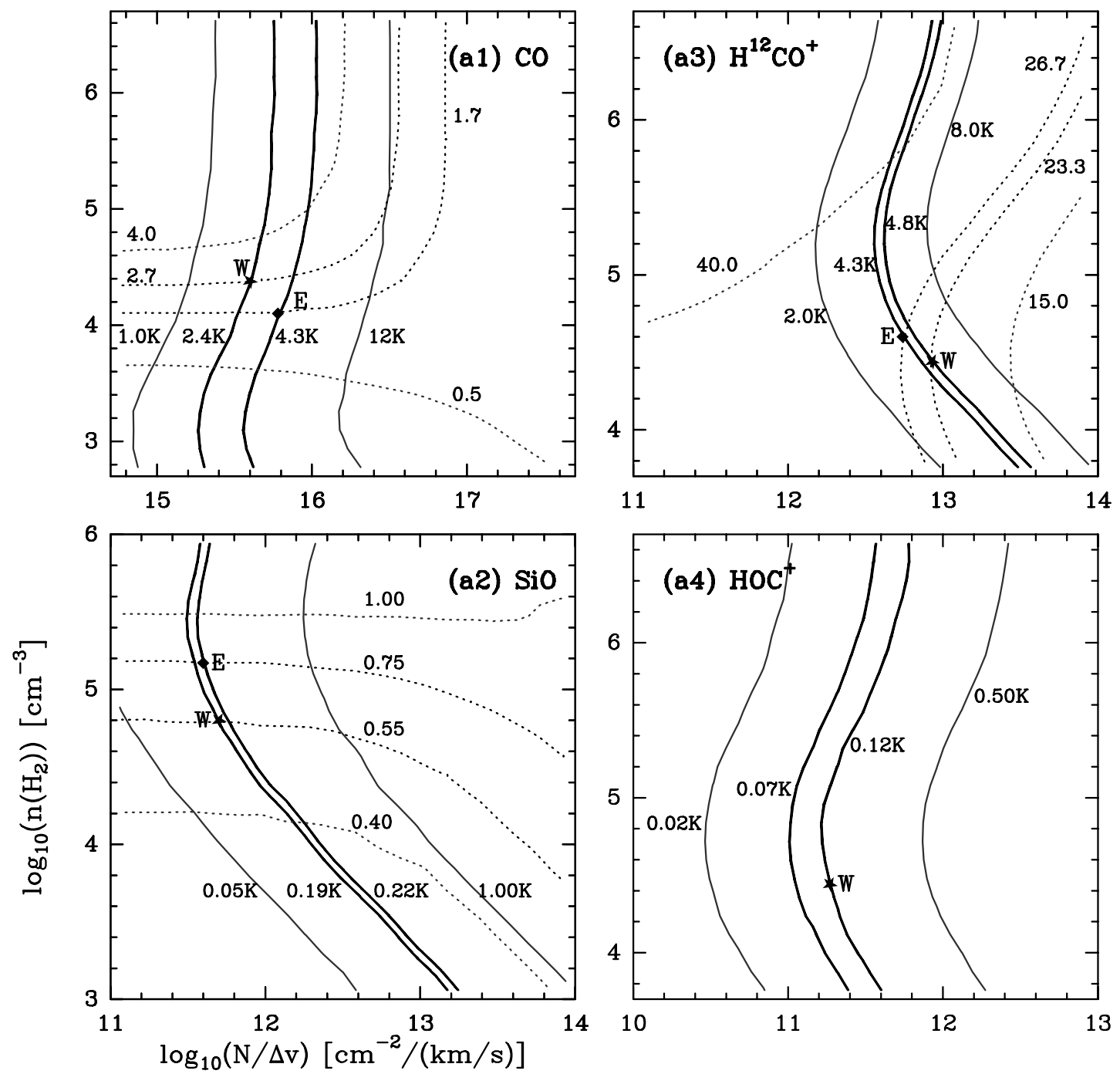

Fig. 6. LVG estimates for oxygenated species in the E/W knots of the CND. a1) For CO, continuous (pointed) curves are contours of constant 1-0 line temperature ((4-3)-to-(1-0) line ratio). a2) For SiO, same for 2-1 line temperature ((3-2)-to-(2-1) line ratio). a3) For $\mathrm{H}^{12} \mathrm{CO}^{+}$, same for 1-0 line temperature $\left(\left[\mathrm{H}^{12} \mathrm{CO}^{+}\right]-\right.$to- $\left.\mathrm{H}^{13} \mathrm{CO}^{+}\right] 1-0$ line ratio). a4) For $\mathrm{HOC}^{+}$, same for $1-0$ line temperature. Squared (starred) markers show solutions for the East (West) knot.

Figures 6 and 7, and Table 5 summarize the results of LVG calculations for $\mathrm{CO}, \mathrm{HCN}, \mathrm{CS}, \mathrm{CN}, \mathrm{SiO}, \mathrm{HCO}^{+}$ and $\mathrm{HOC}^{+}$. Normalized with respect to $N(\mathrm{CO})$, the column densities of $\mathrm{SiO}, \mathrm{HCO}^{+}$and $\mathrm{HOC}^{+}$(i.e., $N(\mathrm{SiO}) / N(\mathrm{CO})$, $N\left(\mathrm{HCO}^{+}\right) / N(\mathrm{CO})$ and $\left.N\left(\mathrm{HOC}^{+}\right) / N(\mathrm{CO})\right)$ are $\geq 2-3$ larger in the $\mathrm{W}$ knot than in the $\mathrm{E}$ knot. In contrast, $N(\mathrm{HCN}) / N(\mathrm{CO})$ and $N(\mathrm{CS}) / N(\mathrm{CO})$ column density ratios are similar in the two knots within a $25 \%$ uncertainty. These abundance ratios are reflecting the asymmetries of the spectra discussed in Sect. 4.2, suggestive of an uneven processing of molecular gas in the CND.

As a byproduct of LVG models for $\mathrm{CO}$, we have estimated the $X \equiv N\left(\mathrm{H}_{2}\right) / I(\mathrm{CO})$ conversion factor for the molecular gas in the CND. Assuming a range of abundance ratios $[\mathrm{CO}] /\left[\mathrm{H}_{2}\right] \sim 5 \times 10^{-5}-10^{-4}$, we infer a $X$ value of $3-6 \times 10^{19} \mathrm{~cm}^{-2} /\left(\mathrm{K} \mathrm{km} \mathrm{s}^{-1}\right)$, i.e., $\sim 4-8$ times smaller than the canonical value $X=2.2 \times 10^{20}$ (Solomon \& Barrett 1991). Unless $\mathrm{CO}$ is underabundant by a similar factor (a scenario invoked by Sternberg et al. 1994 in the oxygen depletion models clearly invalidated by the results of our work; see Sect. 5) we conclude that the $X$ conversion factor is lower in the CND of NGC 1068. Similar deviations have been previously reported in other galactic central regions (Dahmen et al. 1998, and references therein). This might reflect the failure of some of the basic hypothesis that support the canonical value. In particular, the strong gravitational forces near galactic nuclei may prevent molecular clouds from reaching virialization.

\section{Chemistry of molecular gas in the CND of NGC 1068}

To give further insight into the chemistry of molecular gas in the CND we have compared, for a common set of abundance ratios, the values measured in NGC 1068 with those observed in a reference galactic region. Here we take as "zeropoint" environment the "Extended Ridge" of OMC-1 (OER) 

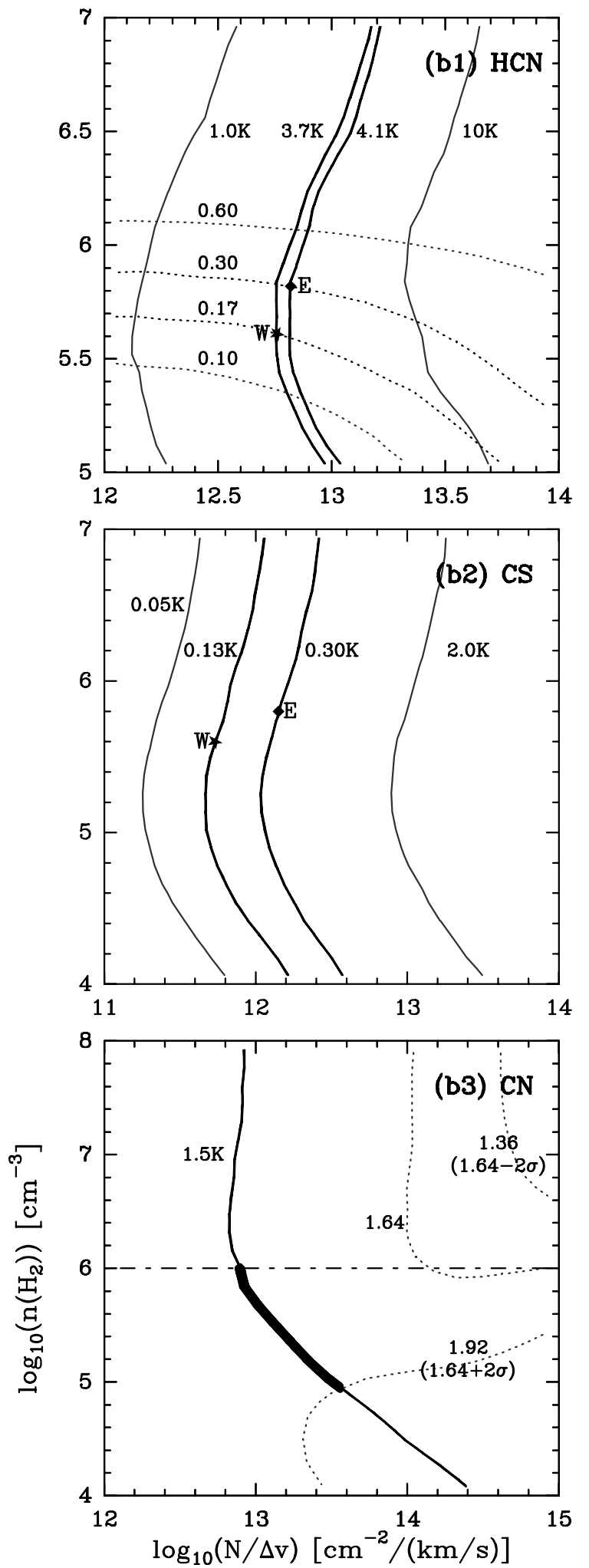

Fig. 7. LVG estimates for non-oxygenated species in the E/W knots of the CND. b1) For HCN, continuous (pointed) lines are contours of constant 1-0 line temperature ((4-3)-to-(1-0) line ratio). b2) For CS, same for 2-1 line temperature. b3) For $\mathrm{CN}$, same for 2-1/high freq. line temperature ((2-1/high freq.)-to-(2-1/low freq.) ratio); a range of possible solutions found is highlighted in bold face: we impose $n\left(\mathrm{H}_{2}\right)<10^{6} \mathrm{~cm}^{-3}$ for consistency with the results from others species and allow for $\mathrm{a} \pm 2 \sigma$ uncertainty in the measured ratio. Markers are as is Fig. 6.
Table 5. LVG results: Col. 1 = chemical species; Col. 2 = parameters determined from the LVG models ( $n$ : molecular gas densities in $\mathrm{cm}^{-3}$; $N / \Delta v$ : column densities per velocity interval in $\mathrm{cm}^{-2} \mathrm{~km}^{-1} \mathrm{~s} ; X$ : chemical abundances relative to $\mathrm{H}_{2}$; we assume $X(\mathrm{CO})=8 \times 10^{-5}$ and compute the rest of abundances accordingly from column density ratios relative to $\mathrm{CO}$ ); Col. 3 = solutions for the East-knot; Col. $4=$ same for the West-knot; Col. 5 = east-to-west ratio of abundances.

\begin{tabular}{|c|c|c|c|c|}
\hline Species & LVG-sol. & E/blue & $\mathrm{W} / \mathrm{red}$ & $N_{\mathrm{E} / \mathrm{W}}$ \\
\hline $\mathrm{CO}$ & $\begin{array}{l}n \\
N / \Delta v \\
X\end{array}$ & $\begin{array}{l}1.3 \times 10^{4} \\
6.3 \times 10^{15} \\
8.0 \times 10^{-5}\end{array}$ & $\begin{array}{l}2.5 \times 10^{4} \\
4.0 \times 10^{15} \\
8.0 \times 10^{-5}\end{array}$ & 1.0 \\
\hline $\mathrm{SiO}$ & $\begin{array}{l}n \\
N / \Delta v \\
X\end{array}$ & $\begin{array}{l}1.6 \times 10^{5} \\
4.0 \times 10^{11} \\
5.1 \times 10^{-9}\end{array}$ & $\begin{array}{l}6.0 \times 10^{4} \\
5.0 \times 10^{11} \\
1.0 \times 10^{-8}\end{array}$ & 0.5 \\
\hline $\mathrm{HCO}^{+}$ & $\begin{array}{l}n \\
N / \Delta v \\
X\end{array}$ & $\begin{array}{l}4.0 \times 10^{4} \\
5.0 \times 10^{12} \\
6.3 \times 10^{-8}\end{array}$ & $\begin{array}{l}2.5 \times 10^{4} \\
8.0 \times 10^{12} \\
1.6 \times 10^{-7}\end{array}$ & 0.4 \\
\hline $\mathrm{HOC}^{+}$ & $\begin{array}{l}n \\
N / \Delta v \\
X\end{array}$ & $\begin{array}{l}- \\
- \\
-\end{array}$ & $\begin{array}{l}2.5 \times 10^{4} \\
2.0 \times 10^{11} \\
4.0 \times 10^{-9}\end{array}$ & - \\
\hline $\mathrm{HCN}$ & $\begin{array}{l}n \\
N / \Delta v \\
X\end{array}$ & $\begin{array}{l}6.3 \times 10^{5} \\
6.3 \times 10^{12} \\
8.0 \times 10^{-8}\end{array}$ & $\begin{array}{l}4.0 \times 10^{5} \\
5.0 \times 10^{12} \\
1.0 \times 10^{-7}\end{array}$ & 0.8 \\
\hline CS & $\begin{array}{l}n \\
N / \Delta v \\
X\end{array}$ & $\begin{array}{l}6.3 \times 10^{5} \\
1.6 \times 10^{12} \\
2.0 \times 10^{-8}\end{array}$ & $\begin{array}{l}4.0 \times 10^{5} \\
8.0 \times 10^{11} \\
1.6 \times 10^{-8}\end{array}$ & 1.2 \\
\hline $\mathrm{CN}$ & $\begin{array}{l}n \\
N / \Delta v \\
X\end{array}$ & $\begin{array}{r}\text { (global } \\
>1.6 \\
1-5 \\
9 \times 10^{-8}\end{array}$ & $\begin{array}{l}\text { values) } \\
<10^{5} \\
10^{13} \\
5 \times 10^{-7}\end{array}$ & - \\
\hline
\end{tabular}

(Blake et al. 1987), a relatively quiescent molecular region whose chemistry has been described as intermediate between the one typical of cold dark clouds and that of warm cores (Sutton et al. 1995). The choice of the OER as a reference region is also motivated by the similarity of physical parameters of molecular gas density $\left(n\left(\mathrm{H}_{2}\right) \sim 10^{4}-10^{5} \mathrm{~cm}^{-3}\right)$ and kinetic temperature $\left(T_{\mathrm{K}} \sim 50 \mathrm{~K}\right)$ in the CND and in the OER. Therefore, significant differences in the abundance ratios of "critical" tracers between the CND and the OER can be mostly attributed to different chemistries being at work in these regions. We will also use the OER as the "zero point" basis to extrapolate the abundance ratios in the case of oxygen depletion models (Ruffle et al. 1998).

We list in Table 6 the following set of abundance ratios: $N(\mathrm{HCN}) / N(\mathrm{CO}), N(\mathrm{CS}) / N(\mathrm{CO}), N(\mathrm{HCN}) / N\left(\mathrm{HCO}^{+}\right)$, $N(\mathrm{CN}) / N(\mathrm{HCN}), N(\mathrm{SiO}) / N(\mathrm{CO})$ and $N\left(\mathrm{HCO}^{+}\right) / N\left(\mathrm{HOC}^{+}\right)$. These abundance ratios can be significantly different depending on the chemical environment. As argued below, an evaluation of these ratios allows us to compare the chemical status of the CND and the OER with the predictions of models invoking either oxygen-depletion or X-ray driven chemistry:

- Our observations provide new constraints for oxygendepletion models first proposed by Sternberg et al. (1994) 
Table 6. Abundance ratios predicted/observed in different molecular regions: the E-knot of the CND of NGC 1068, same for the West-knot; a prototypical XDR (Lepp \& Dalgarno 1996; Yan \& Dalgarno 1997), the Orion Extended Region (OER) (Blake et al. 1987), and the OER corrected with oxygen depletion (Ruffle et al. 1998).

\begin{tabular}{|c|c|c|c|c|c|}
\hline Abundance ratios & $\mathrm{CND}(\mathrm{E} / \mathrm{blue})$ & $\mathrm{CND}(\mathrm{W} / \mathrm{red})$ & XDR & OER & $\begin{array}{l}\text { OER+oxygen depletion } \\
\text { (Gas phase model) }\end{array}$ \\
\hline $\mathrm{HCN} / \mathrm{CO}$ & $1.0 \times 10^{-3}$ & $1.2 \times 10^{-3}$ & $5 \times 10^{-4}$ & $1.0 \times 10^{-4}$ & $1 \times 10^{-3}$ \\
\hline $\mathrm{CS} / \mathrm{CO}$ & $2.5 \times 10^{-4}$ & $1.6 \times 10^{-4}$ & $1-5 \times 10^{-4}$ & $5.0 \times 10^{-5}$ & $7 \times 10^{-5}$ \\
\hline $\mathrm{HCN} / \mathrm{HCO}^{+}$ & 1.3 & 0.6 & $0.5-1$ & 2.2 & 25 \\
\hline $\mathrm{CN} / \mathrm{HCN}$ & \multicolumn{2}{|c|}{$1-5$ (global value $)$} & 3 & 0.7 & 20 \\
\hline $\mathrm{SiO} / \mathrm{CO}$ & $6.4 \times 10^{-5}$ & $1.2 \times 10^{-4}$ & - & $<6.6 \times 10^{-6}$ & - \\
\hline $\mathrm{HCO}^{+} / \mathrm{HOC}^{+}$ & - & 50 & - & - & - \\
\hline
\end{tabular}

as an explanation for the high $\mathrm{HCN} / \mathrm{CO}$ ratio measured in the CND of NGC 1068. This scenario is supported by $\mathrm{X}$-ray and ultraviolet observations of the hot-ionized gas in the narrow-line region of NGC 1068 (Marshall et al. 1993; Ogle et al. 2003). With the inclusion of dust-grain chemistry in time-dependent models, Shalabiea \& Greenberg (1996) were able to fit at "early times" ( $t \simeq 10^{6} \mathrm{yr}$ ) $\mathrm{HCN} / \mathrm{CO} \sim$ a few $10^{-3}$ with values less restrictive for the oxygen depletion. The overall consequences of selective oxygen-depletion in the chemistry of molecular clouds have been more extensively studied in the framework of gas-phase (Terzieva et al. 1998; Ruffle et al. 1998) and gas-grain chemical models (Shalabiea 2001). The primary effect of an oxygen underabundance is a reduced formation of $\mathrm{CO}$. The fraction of carbon not consumed in the $\mathrm{CO}$ synthesis is then increased and it can thus enhance the abundances of some carbonated species, such as HCN, $\mathrm{CS}$ or $\mathrm{CN}$; on the contrary, abundances of oxygen-bearing species are expected to be lower. This decrease is less important for $\mathrm{HCO}^{+}$, as in this case a lower oxygen abundance is mostly balanced by the increase of available carbon.

As shown in Table 6, the measured $\mathrm{HCN} / \mathrm{CO}$ ratio in the CND of NGC 1068 ( a few $10^{-3}$ ) is 1 order of magnitude larger than that derived for the OER. Oxygen depletion models can fit the HCN-to-CO ratio of the CND with an oxygen depletion of $[\mathrm{O}]_{\mathrm{CND}} /[\mathrm{O}]_{\mathrm{OER}} \sim 1 / 2$. However, this value of oxygen depletion would lead to large $\mathrm{HCN} / \mathrm{HCO}^{+}$ ratios $(\sim 25)$ which are at odds with the low ratios $(\sim 1)$ of the CND. Furthermore, these models predict a significant enhancement of $\mathrm{CN}$ due to the reduction of $\mathrm{O}$ I which is an important source of $\mathrm{CN}$ destruction (Bachiller et al. 1997). Here also the CN-to-HCN ratio in the oxygen depletion models solution $(\sim 20)$ is nearly one order of magnitude larger than the CND ratio $(\sim 1-5$, i.e., slightly above the OER standards). Finally, the predicted variation for the $\mathrm{CS} / \mathrm{CO}$ ratio is marginal $(\times 1.4)$ in the adopted oxygendepletion solution, leading to values similar to that reported for the CND: $\mathrm{CS} / \mathrm{CO} \sim 2 \times 10^{-4}$.

- Lepp \& Dalgarno (1996) proposed an alternative explanation of the high $\mathrm{HCN} / \mathrm{CO}$ ratio measured in the CND of NGC 1068: X-rays coming from the central engine may significantly enhance the abundance of $\mathrm{HCN}$ in the neighbouring molecular gas. Thus, the $\mathrm{HCN} / \mathrm{CO}$ ratio measured in NGC 1068 can be easily accounted for. In a XDR chemistry some diatomic species, such as $\mathrm{CN}$ and $\mathrm{OH}$ are particularly robust (Lepp \& Dalgarno 1996). Moreover, large abundances of $\mathrm{OH}$ favour the formation of $\mathrm{CO}^{+}$and $\mathrm{H}_{2} \mathrm{O}$ (Sternberg et al. 1996); these species take part directly in the production of large quantities of $\mathrm{HCO}^{+}$. The abundances of $\mathrm{HCN}, \mathrm{CN}$ and $\mathrm{HCO}^{+}$simultaneously reach their peak values at similar depths inside XDR (Yan \& Dalgarno 1997). The XDR model of Yan \& Dalgarno (1997) predicts an average $\mathrm{CS} / \mathrm{CO}$ abundance ratio of $1-5 \times 10^{-4}$ for the range of depths inside the XDR that are expected to dominate the emission of molecular gas. As summarized in Table 6, the $\mathrm{HCN} / \mathrm{CO}, \mathrm{HCN} / \mathrm{HCO}^{+}, \mathrm{CN} / \mathrm{HCN}$ and $\mathrm{CS} / \mathrm{CO}$ abundance ratios predicted by XDR models (see Lepp \& Dalgarno 1996 for the three first ratios; the CS/CO ratio has been estimated from Yan \& Dalgarno 1997) are in close agreement with the corresponding values estimated for the CND of NGC 1068.

In summary, while oxygen depletion models are able to fit the $\mathrm{HCN} / \mathrm{CO}$ ratio measured in the CND of NGC 1068, the adopted solution leads to $\mathrm{HCN} / \mathrm{HCO}^{+}$and $\mathrm{CN} / \mathrm{HCN}$ abundance ratios which are excessively large compared to that actually measured for the CND. In contrast, the models invoking XDR chemistry explain naturally the ratios measured in NGC 1068; these values depart significantly from the standard reference values of the OER. In the following sections we discuss how the detection of high abundances of $\mathrm{SiO}$ and $\mathrm{HCO}^{+}$in the CND of NGC 1068 adds supporting evidence to the XDR chemistry scenario.

\section{1. $\mathrm{SiO}$ in $\mathrm{XDR}$}

As is shown in Table 6, the $\mathrm{SiO}$-to- $\mathrm{CO}$ abundance ratio measured toward the CND of NGC 1068 is high by normal galactic standards: $N(\mathrm{SiO}) / N(\mathrm{CO}) \sim 6 \times 10^{-5}-1.2 \times 10^{-4}$. The normalized $\mathrm{SiO}$ column densities toward the CND are at least one order of magntitude larger than the upper limit derived for the OER $\left(<7 \times 10^{-6}\right)$. Assuming an absolute abundance for $\mathrm{CO}$ of $X(\mathrm{CO})=8 \times 10^{-5}$, this implies $X(\mathrm{SiO})=5 \times 10^{-9}-1.0 \times 10^{-8}$. As discussed in Sect. 3.1.1, a significant enhancement of $\mathrm{SiO}$ in molecular gas has been attributed to heavy shock processing of grains in starburst galaxies where values of $X(\mathrm{SiO})$ up to a few $10^{-10}$ have been reported on scales of several hundred pc (García-Burillo et al. 2000, 2001). The CND 
abundances of $\mathrm{SiO}$ estimated here are significantly larger than those reported for starbursts, however; this is further evidence that silicon chemistry in the CND is not being driven by star formation. In contrast, the estimated $\mathrm{SiO}$ abundances in the starburst ring of NGC $1068\left(X(\mathrm{SiO}) \sim 2-3 \times 10^{-10}\right)$ are in close agreement with $\mathrm{SiO}$ abundances measured in starbursts on similar spatial scales.

Alternatively, it has been suggested that X-ray irradiated dust grains can enhance silicon chemistry in gas phase. X-rays are able to heat very small silicate grains (10 ̊), subsequently leading to their evaporation and to an enlargement of the Si gasphase fraction (Voit 1991). Most remarkably, the nucleus of NGC 1068 shows a strong Fe K $\alpha$ line (Ogle et al. 2003 and references therein). The bulk of the $6.4 \mathrm{keV}$ line of Fe I most likely comes from fluorescence in the Compton-thick molecular gas torus of NGC 1068. The detection of strong Fe K $\alpha$ line emission is therefore an indication that large column densities of molecular gas are being processed by X-rays. In a precedent study, Martín-Pintado et al. (2000) found a correlation between the intensity of the Fe $6.4 \mathrm{keV}$ line and the derived abundance of $\mathrm{SiO}$ in the $\mathrm{Sgr} \mathrm{A}$ and $\mathrm{Sgr} \mathrm{B}$ molecular complexes at the Galactic Center.

\section{2. $\mathrm{HOC}^{+}$in $\mathrm{XDR}$}

According to the estimates of Sect. $4.3, \mathrm{HOC}^{+}$abundances derived for the CND of NGC 1068 are the largest ever measured in interstellar medium: $X\left(\mathrm{HCO}^{+}\right) / X\left(\mathrm{HOC}^{+}\right) \sim 30-80$. These low ratios are in direct contrast with those typically measured in galactic dense molecular clouds where values from $\sim 6000$ to $\sim 300$ have been reported thus far (Apponi et al. 1997, 1999). Most interestingly, the lowest value found by Apponi et al. (1999) corresponds to the Orion bar, a prototypical PDR. Very low ratios $(\sim 50-120)$ have been recently found in the prototypical PDR NGC 7023 (Fuente et al. 2003). As argued below, we propose that low $R \equiv X\left(\mathrm{HCO}^{+}\right) / X\left(\mathrm{HOC}^{+}\right)$ratios can be explained for molecular clouds with high ionization degrees, either in XDR or in PDR.

The fast hydrogen-catalyzed isomerization of $\mathrm{HOC}^{+}$into $\mathrm{HCO}^{+}$usually shifts the equilibrium between both species towards significantly lower abundances of $\mathrm{HOC}^{+}$. However, as suggested by Smith et al. (2002), the isomerization process converting $\mathrm{HOC}^{+}$into $\mathrm{HCO}^{+}$could be compensated by the destruction of $\mathrm{HCO}^{+}$due to interaction with electrons. This process is likely to be enhanced at high electron densities $\left(X\left(\mathrm{e}^{-}\right) \sim 10^{-5}\right)$. The latter could explain why the lowest $R$ values have been measured in galactic PDR (Apponi et al. 1999; Fuente et al. 2003). Furthermore, the $X\left(\mathrm{HCO}^{+}\right) / X\left(\mathrm{HOC}^{+}\right)$ratio at equilibrium is also sensitive to the dominant mechanism of $\mathrm{HCO}^{+} / \mathrm{HOC}^{+}$formation: the more efficient is the relative production of $\mathrm{HOC}^{+}$, the lower is the ionization degree required to reach a certain $R$ ratio. Typical paths for the formation of $\mathrm{HCO}^{+} / \mathrm{HOC}^{+}$are (Apponi et al. 1997 and references therein):

$$
\begin{aligned}
& \mathrm{H}_{3}^{+}+\mathrm{CO} \longrightarrow \mathrm{HOC}^{+} / \mathrm{HCO}^{+}+\mathrm{H}_{2} \\
& \mathrm{CO}^{+}+\mathrm{H}_{2} \longrightarrow \mathrm{HOC}^{+} / \mathrm{HCO}^{+}+\mathrm{H} \\
& \mathrm{H}_{2} \mathrm{O}+\mathrm{C}^{+} \longrightarrow \mathrm{HOC}^{+} / \mathrm{HCO}^{+}+\mathrm{H} .
\end{aligned}
$$

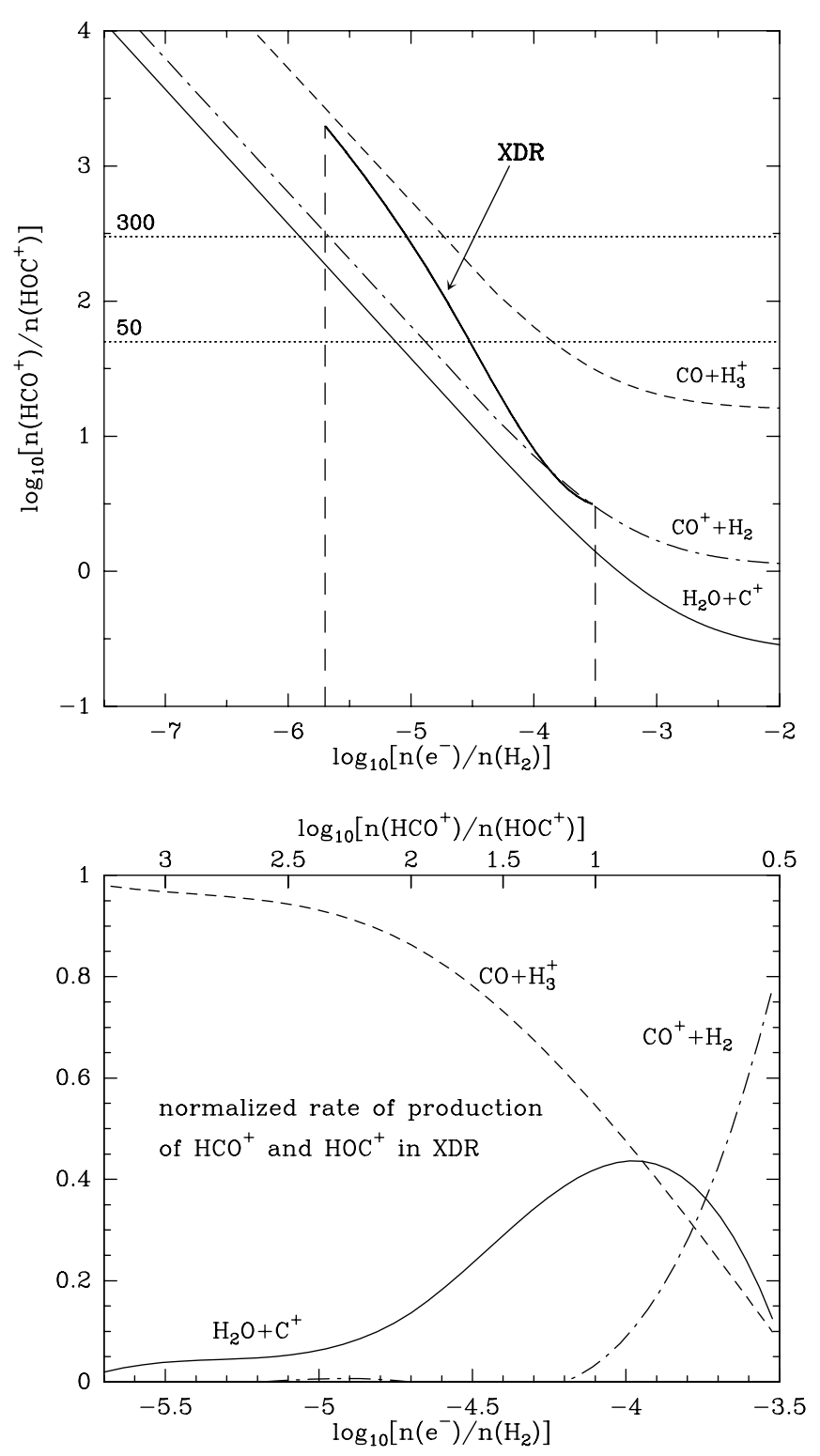

Fig. 8. Top panel: steady state $\mathrm{HCO}^{+}$-to- $\mathrm{HOC}^{+}$abundance ratio as a function of the ionization degree of molecular gas. Curves for single formation paths are plotted; the thick line shows the predicted ratio for a XDR chemistry. Bottom panel: fraction of $\mathrm{HCO}^{+}$and $\mathrm{HOC}^{+}$molecules formed along each chemical path in a XDR chemistry.

The branching ratio for the net production of nascent $\mathrm{HOC}^{+}$, hereafter denoted by $\alpha$, depends on the particular formation pathway. The value of $\alpha$ is 0.06 for reaction 2, 0.48 for 3 and 0.8 for 4 . In a real case scenario the three reactions coexist, and thus the equivalent branching ratio, $\alpha_{\text {eff }}$, is an average of the individual $\alpha$, weighted by the fraction of $\mathrm{HCO}^{+}$and $\mathrm{HOC}^{+}$ particles that are actually formed following a certain pathway. We have derived how $R$ depends on the ionization degree of molecular gas, separately, for the different reactions, assuming the rate coefficients given by Smith et al. (2002) (Fig. 8, top panel). We find that in order to obtain values of $R \sim 50-300$ high ionization degrees are needed: $X\left(\mathrm{e}^{-}\right) \sim 10^{-6}-10^{-4}$. These high electronic abundances are typically reached in XDR 


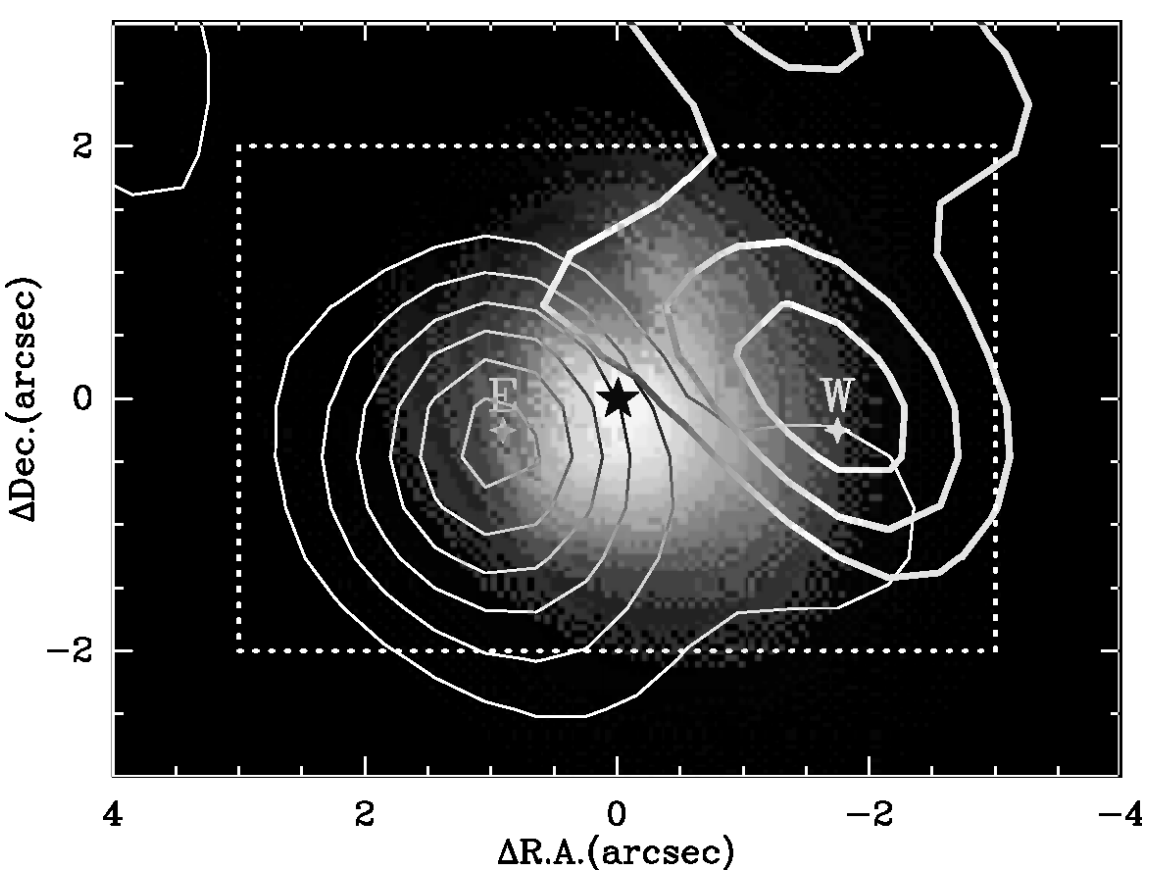

Fig. 9. X-ray emission and molecular gas in the CND: overlay of the distribution of hard $\mathrm{X}$-ray emission in the 6-8 $\mathrm{keV}$ band (gray scale adapted from Ogle et al. 2003: whiter shades stand for stronger emission) and the $\mathrm{CO}(1-0)$ integrated emission as in Fig. 3. The AGN locus is highlighted by the starred marker.
(Lepp \& Dalgarno 1996; Maloney et al. 1996). Figure 8 illustrates also the relation between the dominant formation path and the isomer ratio: less extreme ionization degrees are needed to reach low $R$ ratios if the predominant reaction has a large $\alpha$. On the other hand, much higher ratios $\sim 300-6000$, like that typically measured in molecular clouds, can be easily accounted for if electronic abundances approach standard levels $<10^{-6}-10^{-7}$.

We have also derived the dependence of $\alpha_{\text {eff }}$ on the ionization degree for an adopted XDR model (see the curve for $R$ in the top panel of Fig. 8). The abundances of all molecular species, contributing to (2)-(4), have been taken from Maloney et al. (1996), except for $\mathrm{CO}^{+}$, whose abundance curve is taken from the PDR model of Sternberg et al. (1995). Values of $R \sim 30-80$, like that measured in the CND of NGC 1068, can be easily accounted for assuming an average ionization degree of $X\left(\mathrm{e}^{-}\right) \sim 10^{-5}$ for the bulk of molecular gas.

The relative weight of the 3 formation paths of $\mathrm{HOC}^{+}$in a typical XDR is also represented, as a function of $X\left(\mathrm{e}^{-}\right)$, in Fig. 8 (bottom panel). While reaction (2) clearly dominates the balance for $X\left(\mathrm{e}^{-}\right)<10^{-5}$, reactions involving $\mathrm{H}_{2} \mathrm{O}$ (4) and $\mathrm{CO}^{+}$(3) are predominant for $X\left(\mathrm{e}^{-}\right)>10^{-4}$. Recent observations of galactic PDR (Fuente et al. 2003; Rizzo et al. 2003) have confirmed that low $\mathrm{HCO}^{+} / \mathrm{HOC}^{+}$ratios are indeed correlated with large abundances of $\mathrm{CO}^{+}$and/or $\mathrm{H}_{2} \mathrm{O}$.

\subsection{Anisotropic X-ray illumination of the CND?}

The results of this work strongly favour an overall scenario where the CND of NGC 1068 has become a giant XDR. It is tempting to speculate if X-ray driven chemistry can also explain the mild but systematic differences in the molecular abundances of $\mathrm{SiO}, \mathrm{HCO}^{+}, \mathrm{HOC}^{+}$and $\mathrm{HCN}$ between the $\mathrm{E}$ and the $\mathrm{W}$ knots of the CND. Figure 9 shows the distribution of X-ray emission inside the $6-8 \mathrm{keV}$ band in the CND of NGC 1068 (adapted from Ogle et al. 2003). X-ray emission in this energy band is dominated by the Fe I K $\alpha$ line at $6.4 \mathrm{keV}$ in NGC 1068. There is a $10^{\prime \prime}$ extended emission (not shown in Fig. 9) which corresponds to the ionization cones. The strongest component, however (shown in Fig. 9), should be tracing the illuminated inner wall of the CND torus. Most interestingly, this figure shows tantalizing evidence of a different degree of penetration of X-rays into the E/W knots: the western side of the molecular torus, corresponding to the inner wall of $\mathrm{W}$ knot, seems to be more illuminated than its eastern counterpart. This would be in agreement with the reported chemical differentiation seen between the E/W molecular knots. A difference in the attenuating column densities, estimated from $\mathrm{CO}$, exists between the two CND knots: on $100 \mathrm{pc}$ scales, $\left.N\left(\mathrm{H}_{2}\right)\right|_{\text {East }} /\left.N\left(\mathrm{H}_{2}\right)\right|_{\text {west }} \sim 2$. However, we do not know if these or even greater differences hold at smaller scales which are probably more relevant to probe X-ray absorption by neutral gas in the torus. In this context, it is however suggestive to note that the strongest $\mathrm{H}_{2} \mathrm{O}$ megamasers, which are collisionally excited in the warmest region of the CND illuminated by X-rays (Neufeld et al. 1994), are mostly located in the western side of the molecular torus (Greenhill \& Gwinn 1997).

High-resolution interferometer observations will give a sharp view of molecular abundance changes inside the CND at small scales for critical tracers such as $\mathrm{SiO}, \mathrm{CN}$ and $\mathrm{HOC}^{+}$. A detailed comparison of these maps with the Chandra images of the CND may help to constrain this scenario.

\section{Conclusions}

We summarize the main results obtained in this work as follows:

- We report on the detection of significant $\mathrm{SiO}(3-2)$ and $\mathrm{SiO}(2-1)$ emission in the 200 pc circumnuclear disk of 
NGC 1068. The large overall abundance of $\mathrm{SiO}$ in the CND $\left(\sim(5-10) \times 10^{9}\right)$ cannot be explained by shocks driven by star formation on molecular gas as there is counterevidence of a recent starburst in the nucleus of NGC 1068. While $\mathrm{SiO}$ emission is also detected over the starburst ring, we estimate that $\mathrm{SiO}$ abundances there are 10 times lower than those measured in the CND. These lower abundances of $\mathrm{SiO}$ are in close agreement with that measured in starbursts on similar spatial scales, however.

- We also report on the first extragalactic detection of the reactive ion $\mathrm{HOC}^{+}$. Most remarkably, the estimated $\mathrm{HCO}^{+} / \mathrm{HOC}^{+}$abundance ratio in the nucleus of NGC 1068 , $\sim 30-80$, is the smallest ever measured in molecular gas. The line profile of $\mathrm{HOC}^{+}$is markedly asymmetrical with respect to $v_{\text {sys }}$ : $\mathrm{HOC}^{+}$emission is mostly detected at red velocities. Whatever process is responsible for the enhancement of this reactive ion, it seems to be unevenly efficient inside the CND.

- Results from additional mm-observations have served for estimating abundances of $\mathrm{CN}, \mathrm{HCO}^{+}, \mathrm{HOC}^{+}, \mathrm{H}^{13} \mathrm{CO}^{+}$ and HCO. These estimates are complemented by a reevaluation of molecular abundances for $\mathrm{HCN}, \mathrm{CS}$ and $\mathrm{CO}$, based on previously published single-dish and interferometer observations of NGC 1068. While models invoking oxygen depletion in molecular gas successfully fit the $\mathrm{HCN} / \mathrm{CO}$ ratio measured in the $\mathrm{CND}$, they fail to account for our estimates of the $\mathrm{HCN} / \mathrm{HCO}^{+}$and $\mathrm{CN} / \mathrm{HCN}$ abundance ratios. On the contrary, XDR models can simultaneously explain these ratios. The detection of high abundances of $\mathrm{SiO}$ and $\mathrm{HOC}^{+}$in the CND of NGC 1068 gives further support to the XDR chemistry scenario. The processing of $10 \AA$ dust grains by $\mathrm{X}$-rays, as a mechanism to enhance silicon chemistry in gas phase, would explain the large $\mathrm{SiO}$ abundances of the CND. Finally, we have shown that the low $\mathrm{HCO}^{+} / \mathrm{HOC}^{+}$ratios measured in the CND can be explained if molecular clouds have the high ionization degrees typical of $\operatorname{XDR}\left(X\left(\mathrm{e}^{-}\right) \sim 10^{-6}-10^{-4}\right)$. An examination of the different formation paths of $\mathrm{HOC}^{+}$suggests that reactions involving $\mathrm{H}_{2} \mathrm{O}$ and/or $\mathrm{CO}^{+}$would be the predominant precursors of $\mathrm{HOC}^{+}$in XDR.

- The XDR scenario could also provide an explanation for the different abundances of $\mathrm{SiO}, \mathrm{HCO}^{+}$and, especially, of $\mathrm{HOC}^{+}$measured in the $\mathrm{E}$ and $\mathrm{W}$ knots. The Chandra images of the CND in the $6-8 \mathrm{keV}$ band, dominated by the emission of the Fe I K $\alpha$ line, show tantalizing evidence of a different degree of penetration of hard X-rays into the $\mathrm{E}$ and $\mathrm{W}$ knots. This suggests that larger columns of molecular gas are being processed by X-rays in the $\mathrm{W}$ knot.

Acknowledgements. We acknowledge the IRAM staff from Pico Veleta and Granada for help provided during the observations. We wish to thank A. Rodríguez-Franco for his support during the observations. We also wish to thank E. Schinnerer and L. J. Tacconi for providing their interferometer data. This research has made use of NASA's Astrophysics Data System (ADS) and the NASA/IPAC Extragalactic Database (NED). This paper has been partially funded by the Spanish MCyT under projects DGES/AYA2000-0927, ESP2001-4519-PE, ESP2002-01693, PB1998-0684, ESP2002-01627 and AYA2002-10113E.

\section{References}

Apponi, A. J., Pesch, T. C., \& Ziurys, L. M. 1999, ApJ, 519, L89

Apponi, A. J., \& Ziurys, L. M. 1997, ApJ, 481, 800

Bachiller, R., Fuente, A., Bujarrabal, V., et al. 1997, A\&A, 319, 235

Blake, G. A., Sutton, E. C., Masson, C. R., \& Phillips, T. G. 1987, ApJ, 315, 621

Black, J. H. 1998a, Faraday Discuss., 109, 257

Black, J. H. 1998b, in The Molecular Astrophysics of Stars and Galaxies, ed. T. W. Hartquist, \& D. A. Williams (Oxford: Oxford Univ. Press), 469

Bland-Hawthorn, J., Gallimore, J. F., Tacconi, L. J., et al. 1997, Ap\&SS, 248, 9

Cid-Fernandes, R., Heckman, T., Schmitt, H., González-Delgado, R. M., \& Storchi-Bergmann, T. 2001, ApJ, 558, 81

Davies, R. I., Sugai, H., \& Ward, M. J. 1998, MNRAS, 300, 388

Dahmen, G., Hüttemeister, S., Wilson, T. L., \& Mauersberger, R. 1998, A\&A, 331, 959

Fuente, A., Black, J. H., Martín-Pintado, J., et al. 2000, ApJ, 545, L113

Fuente, A., Rodríguez-Franco, A., García-Burillo, S., Martín-Pintado, J., \& Black, J. H. 2003, A\&A, 406, 899

Gallimore, J. F., Baum, S. A., O’Dea, C. P., \& Pedlar, A. 1996a, ApJ, 458,136

Gallimore, J. F., Baum, S. A., \& O'Dea, C. P. 1996b, ApJ, 464, 198

García-Burillo, S., Martín-Pintado, J., Fuente, A., \& Neri, R. 2000, A\&A, 355, 499

García-Burillo, S., Martín-Pintado, J., Fuente, A., \& Neri, R. 2001, ApJ, 563, L27

García-Burillo, S., Martín-Pintado, J., Fuente, A., Usero, A., \& Neri, R. 2002, ApJ, 575, L55

Genzel, R., Lutz, D., Sturm, E., et al. 1998, ApJ, 498, 579

González-Delgado, R. M., Heckman, T., \& Leitherer, C. 2001, ApJ, 546,845

Greenhill, L. J., \& Gwinn, C. R. 1997, Ap\&SS, 248, 261

Helfer, T. T., \& Blitz, L. 1995, ApJ, 450, 90

Imanishi, M. 2002, ApJ, 569, 44

Kohno, K., Matsushita, S., Vila-Vilaró, B., et al. 2001, in The Central kpc of Starbursts and AGN: The La Palma Connection, ed. J. H. Knapen, J. E. Beckman, I. Shlosman, \& T. J. Mahoney, ASP Conf. Ser., 249, 672

Laurent, O., Mirabel, I. F., Charmandaris, V., et al. 2000, A\&A, 359, 887

Lepp, S., \& Dalgarno, A. 1996, A\&A, 306, L21

Maloney, P. R., Hollenbach, D. J., \& Tielens, A. G. G. M. 1996, ApJ, 466, 561

Mao, R. Q., Henkel, C., Schulz, A., et al. 2000, A\&A, 358, 433

Marco, O., \& Brooks, K. J. 2003, A\&A, 398, 101

Marshall, F. E., Netzer, H., Arnaud, K. A., et al. 1993, ApJ, 405, 168

Martín-Pintado, J., de Vicente, P., Rodríguez-Fernández, N. J., Fuente, A., \& Planesas, P. 2000, A\&A, 356, L5

Neufeld, D. A., Maloney, P. R., \& Conger, S. 1994, ApJ, 436, L127

Ogle, P. M., Brookings, T., Canizares, C. R., Lee, J. C., \& Marshall, H. L. 2003, A\&A, 402, 849

Planesas, P., Gómez-Gónzalez, J., \& Martín-Pintado, J. 1989, A\&A, 216,1

Planesas, P., Scoville, N., \& Myers, S. T. 1991, ApJ, 369, 364

Rizzo, J. R., Fuente, A., Rodríguez-Franco, A., \& García-Burillo, S. 2003, ApJ, 597, L153

Ruffle, D. P., Hartquist, T. W., Caselli, P., Rawlings, J. M. C., \& Williams, D. A. 1998, Ap\&SS, 262, 177

Schenewerk, M. S., Snyder, L. E., \& Hjalmarson, A. 1986, ApJ, 303, L71 
Schenewerk, M. S., Snyder, L. E., Hollis, J. M., Jewell, P. R., \& Ziurys, L. M. 1988, ApJ, 328, 785

Schilke, P., Pineau des Forêts, G., Walmsley, C. M., \& Martín-Pintado, J. 2001, A\&A, 372, 291

Schinnerer, E., Eckart, A., Tacconi, L. J., Genzel, R., \& Downes, D. 2000, ApJ, 533, 850

Scoville, N. Z., Matthews, K., Carico, D. P., \& Sanders, D. B. 1988, ApJ, 327, L61

Shalabiea, O. M., \& Greenberg, J. M. 1996, A\&A, 307, 52

Shalabiea, O. M. 2001, A\&A, 370, 1044

Smith, M. A., Schlemmer, S., von Richthofen, J., \& Gerlich, D. 2002, ApJ, 578, L87

Solomon, P. M., \& Barrett, J. W. 1991, in Proc. 146th Symp. IAU, Dynamics of Galaxies and Their Molecular Cloud Distributions, ed. F. Combes, \& F. Casoli (Dordrecht: Kluwer Academic Publishers), 235
Sternberg, A., \& Dalgarno, A. 1995, ApJS, 99, 565

Sternberg, A., Genzel, R., \& Tacconi, L. J. 1994, ApJ, 436, L131

Sternberg, A., Yan, M., \& Dalgarno, A. 1996, in Molecules in Astrophysics: Probes and Processes, ed. E. F. van Dishoeck (Dordrecht: Kluwer), IAU Symp., 178, 141

Sutton, E. C., Peng, R., Danchi, W. C., et al. 1995, ApJS, 97, 455

Tacconi, L. J., Gallimore, J. F., Genzel, R., Schinnerer, E., \& Downes, D. 1997, Ap\&SS, 248, 59

Tacconi, L. J., Genzel, R., Blietz, M., et al. 1994, ApJ, 426, L77

Terzieva, R., \& Herbst, E. 1998, ApJ, 501, 207

Thatte, N., Quirrenbach, A., Genzel, R., Maiolino, R., \& Tecza, M. 1997, ApJ, 490, 238

Voit, G. M. 1991, ApJ, 379, 122

Wannier, P. G. 1980, ARA\&A, 18, 399

Yan, M., \& Dalgarno, A. 1997, ApJ, 481, 296 